\title{
Fast, Multicore-Scalable, Low-Fragmentation Memory Allocation through Large Virtual Memory and Global Data Structures
}

\author{
Martin Aigner Christoph M. Kirsch Michael Lippautz \\ University of Salzburg, Austria \\ firstname.lastname@cs.uni-salzburg.at
}

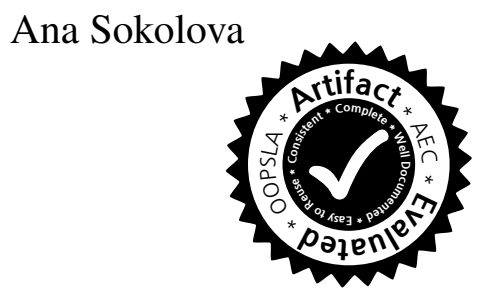

\begin{abstract}
We demonstrate that general-purpose memory allocation involving many threads on many cores can be done with high performance, multicore scalability, and low memory consumption. For this purpose, we have designed and implemented scalloc, a concurrent allocator that generally performs and scales in our experiments better than other allocators while using less memory, and is still competitive otherwise. The main ideas behind the design of scalloc are: uniform treatment of small and big objects through so-called virtual spans, efficiently and effectively reclaiming free memory through fast and scalable global data structures, and constant-time (modulo synchronization) allocation and deallocation operations that trade off memory reuse and spatial locality without being subject to false sharing.
\end{abstract}

Categories and Subject Descriptors D.4.2 [Operating Systems]: Storage Management-Allocation/deallocation strategies; D.3.4 [Programming Languages]: ProcessorsMemory management (garbage collection)

Keywords Memory allocator, virtual memory, concurrent data structures, multicore scalability

\section{Introduction}

Dynamic memory management is a key technology for highlevel programming. Most of the existing memory allocators are extremely robust and well designed. Nevertheless, dynamic memory management for multicore machines is still a challenge. In particular, allocators either do not scale in performance for highly dynamic allocation scenarios, or they do scale but consume a lot of memory. Scalable performance of concurrent programs is crucial to utilize multicore hard-

Permission to make digital or hard copies of part or all of this work for personal or classroom use is granted without fee provided that copies are not made or distributed for profit or commercial advantage and that copies bear this notice and the full citation on the first page. Copyrights for third-party components of this work must be honored. For all other uses, contact the Owner/Author.

Copyright is held by the owner/author(s).

OOPSLA'15, October 25-30, 2015, Pittsburgh, PA, USA

ACM. 978-1-4503-3689-5/15/10

http://dx.doi.org/10.1145/2814270.2814294 ware. Scalable allocation is needed for scalable performance of concurrent programs which allocate memory dynamically.

Even though general purpose allocation was shown superior to custom allocation [4] a decade ago, many multithreaded applications still use custom allocation or limit dynamic allocation to very few threads. We have developed a new memory allocator called scalloc that enables programmers to design many-threaded high-performance applications that dynamically allocate memory without the need for any additional application-tailored allocation strategies.

There are three high-level challenges in the design of a competitive concurrent allocator: (1) fast allocation for high performance, (2) memory layout facilitating fast access, and (3) effective and efficient reuse of memory for low memory consumption. All need to be achieved for various object sizes. Moreover, in presence of concurrency, all need to be achieved with as little coordination as possible. In particular, it is well-established common practice in concurrent allocators to allocate from thread-local allocation buffers (TLABs) [3] for fast allocation. The memory layout needs to support spatial locality without introducing false sharing. Finally, the key challenge is the reuse of free memory: When many threads deallocate objects of various sizes, even in other threads' TLABs, the freed memory should ideally be globally available for immediate reuse by any thread (requiring thread synchronization) for any request (requiring memory defragmentation). Coordination is necessary for memory reuse, the challenge is to minimize its need and cost.

Most competitive concurrent allocators are separated into a local (TLAB-based) frontend, for fast allocation, and a global backend, for reusing memory. TLABs grow and shrink incrementally in chunks of memory which we call spans. Note that spans are called superblocks in Hoard [3], SLABs in llalloc [23], superpages in Streamflow [27], and spans in TCMalloc [10]. Allocation is thread-local and fast as long as there is space in the allocating thread's TLAB, otherwise the TLAB needs to grow by a new span. Therefore, the larger the spans are, the faster the allocation. However, the larger the spans, the higher the memory consumption, since free memory in the TLAB of a given thread is not available to any other thread. Only when a span is empty it can be reused by 
other threads via the global backend, or even be returned to the operating system. The scalability of the global backend is crucial for the scalability of the allocator. Hence, there is a trade-off between scalability and memory consumption.

There are two possibilities in the design of spans: either all spans are of the same size, or not. If not all spans are of the same size, then making free spans reusable for as many requests as possible requires thread synchronization and defragmentation (to control external fragmentation). If they are of the same size, then free spans can be reused by all requests up to the size of the spans, only requiring thread synchronization. The disadvantage of same-sized spans that can accommodate small but also big objects is increased memory consumption (due to internal fragmentation). Most competitive concurrent allocators are therefore hybrids that use relatively small same-sized spans for small objects and different-sized spans for big objects.

To address the challenges in scalable concurrent allocation, we have designed scalloc based on three main ideas introduced here: (a) virtual spans which are same-sized spans in virtual memory; (b) a scalable global backend based on recently developed scalable concurrent data structures; and (c) a constant-time (modulo synchronization) frontend that eagerly returns spans to the backend. We have implemented these three new concepts in scalloc which is open source written in standard $\mathrm{C} / \mathrm{C}++$ and supports the POSIX API of memory allocators (malloc, posix_memalign, free, etc.).

Scalloc provides scalability and reduces memory consumption at the same time. Virtual spans reduce the need and cost of coordination (as they are all of the same size) and at the same time reduce memory consumption. Namely, a virtual span contains a real span of a typically much smaller size for actual allocation. Due to on-demand paging, the rest of the virtual span does not manifest in actual memory consumption. The trick is that the operating system implicitly takes care of physical memory fragmentation because the fraction of the virtual span that is unused has no cost aside of consuming virtual address space. Moreover, the same size of virtual spans allows us to use a single global data structure for collecting and reusing empty spans. Global shared data structures were long considered performance and scalability bottlenecks that were avoided by introducing complex hierarchies. Recent developments in concurrent data structure design show that fast and scalable pools, queues, and stacks are possible $[1,11,15]$. We leverage these results by providing a fast and scalable backend, called the span-pool, that efficiently and effectively reuses and returns free memory to the operating system (through madvise system calls). To the best of our knowledge, no other concurrent allocator uses a single global data structure as its backend. The frontend takes advantage of the scalable backend by eagerly returning spans as soon as they get empty. In contrast to other approaches, the frontend runs per-thread in constant time (modulo synchronization) meaning that at least one thread will make progress in constant time while others may have to retry. The combination of all three design choices is needed to achieve scalability and low memory consumption.

Scalloc is based on a mix of lock-free and lock-based concurrent data structures to minimize code complexity without sacrificing performance (scalloc is implemented in around 3000 lines of code). In our and others' [13] experience locks are still a good choice for synchronizing data structures that are mostly uncontended.

Our experiments show that scalloc increases performance on average while consuming less memory than the previously fastest allocator (TBB). Furthermore, scalloc outperforms and outscales all allocators for varying objects sizes that fit virtual spans while consuming as much or less memory. Scalloc handles spatial locality without being subject to active or passive false sharing, like some other allocators. Access to memory allocated by scalloc is as fast or faster than with most other allocators.

In the following section we provide high-level insight into the experience we gained when developing scalloc that is relevant to a broader audience beyond memory management experts. Section 3 explains the design of virtual spans, the span-pool, and the frontend and how they are integrated in scalloc. Section 4 discusses memory fragmentation and algorithmic complexity of scalloc. Section 5 provides implementation details relevant for performance and reproducibility but optional for understanding the rest of the paper. We discuss related work in Section 6 and present the experimental evaluation in Section 7.

\section{Experience and Relevance}

In our experience with multicore machines dynamic memory management may be a temporal and spatial bottleneck on machines with increasingly many cores and an increasing amount of memory. The challenge is to develop fast, scalable, and low-fragmentation allocators that provide fast memory access and are robust, i.e., do all of that for as many workloads as possible.

The following summary reflects our experience which we obtained in numerous experiments with different configurations and versions of scalloc as well as with many other state-of-the-art allocators.

-64-bit address spaces and on-demand paging help reduce memory consumption and code complexity. On 64-bit machines even extremely large amounts of virtual address fragmentation can be tolerated because of on-demand paging and the sheer number of virtual addresses available. For example, when allocating 1-byte objects only, scalloc in its default configuration may still allocate up to 256GB of physical memory in $32 \mathrm{~TB}$ of virtual memory (16KB real spans in $2 \mathrm{MB}$ virtual spans). As on-demand paging does not map unused virtual memory, defragmentation is only done (simply through unmapping) when resizing empty real spans. Virtual spans enable uniform treatment 
of a large range of object sizes (1-byte to $1 \mathrm{MB}$ objects in default scalloc) only leaving huge objects $(>1 \mathrm{MB})$ to the operating system. In particular, virtual spans enable the use of a single global data structure for collecting and reusing empty spans called the span-pool. However, that data structure still needs to scale in performance.

- Lock-freedom improves performance and scalability but it may not always be necessary for overall performance and scalability. The span-pool is a so-called distributed stack [11] of lock-free Treiber stacks [29] (stacks rather than queues for spatial locality). In default scalloc there are as many Treiber stacks in the span-pool as there are cores on the machine. Access to the span-pool works by first identifying one of the Treiber stacks, which is fast, and then operating on that, possibly in parallel with as many threads as there are cores. The key insight here is that a span-pool with only one Treiber stack does not scale anymore but, most interestingly, results in slightly lower memory consumption because threads find empty spans even faster. Moreover, replacing Treiber stacks with lock-based stacks results in loss of performance and limits scalability [15]. In a different part of scalloc we nevertheless use locks to synchronize access to an uncontended double-ended queue. Here, locking significantly reduces code complexity while not harming overall performance and scalability.

- Constant-time deallocation is not only possible and obviously good for robustness but can also be done to save memory. Allocating objects thread-locally without synchronization is fast and standard practice. Deallocating objects, however, is more difficult as it may happen concurrently in the same span. Encouraged by the performance and scalability of the span-pool, a technical innovation in scalloc is that spans are eagerly inserted into the span-pool as soon as they get empty, replacing possibly non-constant-time cleanup later. It turns out that doing so reduces memory consumption further, again without harming performance and scalability.

On top of the design choices, a careful implementation of all concepts is necessary for competitive performance. We note that when comparing with other allocators, the implementations (and not necessarily the design choices) are being compared. Even a great concept may not perform when poorly implemented.

\section{Virtual Spans, Span-Pool, and Frontend}

This section explains on conceptual level how virtual spans, the span-pool, and the frontend work, and how they are integrated in scalloc.

\subsection{Real Spans and Size Classes}

Like many other allocators, scalloc uses the well-known concept of size classes. A (real) span in scalloc is a contiguous

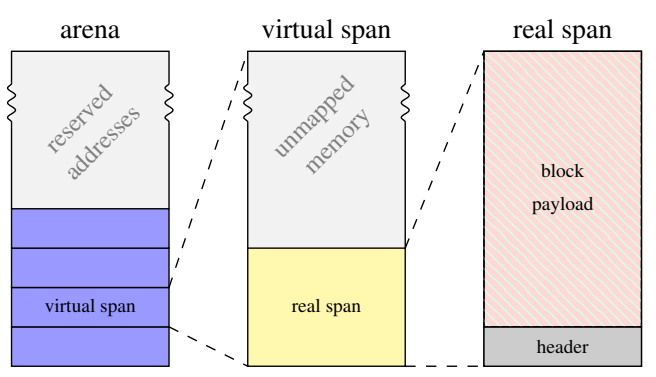

Figure 1: Structure of arena, virtual spans, and real spans

portion of memory partitioned into same-sized blocks. The size of blocks in a span determines the size class of the span. All spans in a given size class have the same number of blocks. In scalloc, there are 29 size classes but only 9 distinct real-span sizes which are all multiples of $4 \mathrm{~KB}$ (the size of a system page).

The first 16 size classes, with block sizes ranging from 16 bytes to 256 bytes in increments of 16 bytes, are taken from TCMalloc [10]. These 16 size classes all have the same real-span size. Size classes for larger blocks range from 512 bytes to $1 \mathrm{MB}$, in increments that are powers of two. These size classes may have different real-span size, explaining the difference between 29 size classes and 9 distinct realspan sizes. The design of size-classes limits block internal fragmentation for sizes larger than 16 bytes to less than $50 \%$.

Objects of size larger than any size class are not managed by spans, but rather allocated directly from the operating system using mmap.

\subsection{Virtual Spans}

A virtual span is a span allocated in a very large portion of virtual memory (32TB) which we call arena. All virtual spans have the same fixed size of $2 \mathrm{MB}$ and are $2 \mathrm{MB}$-aligned in the arena. Each virtual span contains a real span, of one of the available size classes. Wa say "size class of the virtual span" for the size class of the contained real span. Typically, the real span is (much) smaller than the virtual span that contains it. The maximal real-span size is limited by the size of the virtual span. This is why virtual spans are suitable for big objects as well as for small ones. The structure of the arena, virtual spans, and real spans is shown in Figure 1. The advantages of using virtual spans are:

1. Virtual memory outside of real spans does not cause fragmentation of physical memory, as it is not used and therefore not mapped (because of on-demand paging of the operating system);

2. Uniform treatment of small and big objects;

3. No repeated system calls upon every span allocation since the arena is mmapped only once.

Note that since virtual spans are of the same size and aligned in virtual memory, getting a new virtual span from the arena is simply incrementing a bump pointer. When a 
virtual span gets empty, it is inserted into the free-list of virtual spans, i.e., the span-pool discussed in the next section. The disadvantages of using virtual spans are:

1. Current kernels and hardware only provide a 48-bit, instead of a 64-bit, address space. As a result, not all of virtual memory can be utilized (see below);

2. Returning a virtual span to the span-pool may be costly in one scenario: a virtual span with a real span of a given size greater than a given threshold becomes empty and is inserted into the span pool. Then, in order to limit physical-memory fragmentation, we use madvise ${ }^{1}$ to inform the kernel that the remaining virtual (and thus mapped physical) memory is no longer needed.

Note that the design of the span-pool minimizes the chances that a virtual span changes its real-span size.

Mmapping virtual memory in a single call at this order of magnitude (32TB) is a new idea first developed for scalloc. Upon initialization, scalloc mmaps $2^{45}$ virtual memory addresses, the upper limit for a single mmap call on Linux. This call does not introduce any significant overhead as the memory is not mapped by the operating system. It is still possible to allocate additional virtual memory using mmap, e.g. for other memory allocation or memory-mapped I/O. The virtual address space still left is $2^{48}-2^{45}$ bytes, i.e., 224TB.

In the worst case of the current configuration with $2 \mathrm{MB}$ virtual spans, if real spans are the smallest possible (16KB), the physical memory addressable with scalloc is $\left(2^{45} / 2^{21}\right)$. $2^{14}$ bytes $=2^{38}$ bytes $=256 \mathrm{~GB}$.

We have also experimented with configurations of up to $128 \mathrm{MB}$ for virtual spans resulting in unchanged temporal and spatial performance for the benchmarks that were not running out of arena space. Enhancing the Linux kernel to support larger arenas is future work. On current hardware, with up to 48 bits for virtual addresses, this would enable up to 256TB arena space and $2 \mathrm{~TB}$ addressable physical memory (in the worst case, with $2 \mathrm{MB}$ virtual spans and $16 \mathrm{~KB}$ real spans).

Note that in scalloc virtual spans do not restrict the possibility of observing segmentation faults because unmapped memory that is not used by a real-span is still protected against access using the mprotect system call.

\subsection{Backend: Span-Pool}

The span-pool is a global concurrent data structure that logically corresponds to an array of real-span-size-segregated "stack-like" pools. The span-pool implements put and get methods; no values are lost nor invented from thin air; it neither provides a linearizable emptiness check, nor any order guarantees. However, each pool within the span-pool is a locally linearizable [12] "stack-like" pool. It is "stack-like" since in a single-threaded scenario it is actually a stack.

\footnotetext{
${ }^{1}$ madvise is used to inform the kernel that a a range of virtual memory is not needed and the corresponding page frames can be unmapped.
}

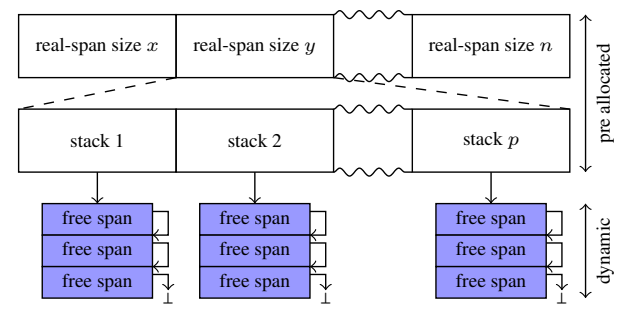

Figure 2: Span-pool layout

Figure 2 illustrates that the segregation by real-span size is implemented as pre-allocated array where each index in the array refers to a given real-span size. Consequently, all size classes that have the same real-span size refer to the same index. Each array entry then holds another pre-allocated array, the pool array, this time of lock-free Treiber stacks [29]. The pool array has size equal to the number of cores (determined at run-time during the initialization phase of the allocator). As a result a stack in any of the pools of the span-pool is identified by a real-span index and a core index. A Treiber stack is a pointer to the top of a singly-linked list of elements; pushing and popping is done lock-free by atomic compareand-swap operations on the top pointer.

The design is inspired by distributed queues [11]. We use stacks rather than queues for the following reasons: spatial locality, especially on thread-local workloads; lower latency of push and pop compared to enqueue and dequeue; and stacks can be implemented without sentinel nodes, i.e., no additional memory is needed for the data structure. Thereby, we utilize the memory of the elements inserted into the pool to construct the stacks, avoiding any dynamic allocation of administrative data structures. Distributed stacks are, to our knowledge, among the fastest scalable pools. To make the occurrence of the ABA problem [16] unlikely we use 16-bit ABA counters that are embedded into link pointers ${ }^{2}$. Completely avoiding the ABA problem is a non-trivial task, which can be solved using e.g. hazard pointers [24].

Listing 1 shows the pseudo code of the span-pool. Upon returning a span to the span-pool, a thread performing a put call first determines the real-span index for a given span (line 21) and the core index as thread identifier modulo the number of cores (line 22). Before actually inserting (line 25) the given span into the corresponding stack the thread may return the span's underlying memory to the operating system using the madvise system call with advice MADV_DONTNEED (line 24), effectively freeing the affected memory. This is the expensive case, only performed on spans with large real-span size determined by a threshold, as unused spans with large physically mapped real-spans result in noticeable physical fragmentation and the madvise system call may anyway be necessary upon later reuse, e.g. when a span is to be reused in a size class with

\footnotetext{
${ }^{2}$ Currently a 64-bit address space is limited to 48 bits of address, enabling the other 16 bits to be used as ABA counter.
} 
Listing 1: Span-pool pseudo code

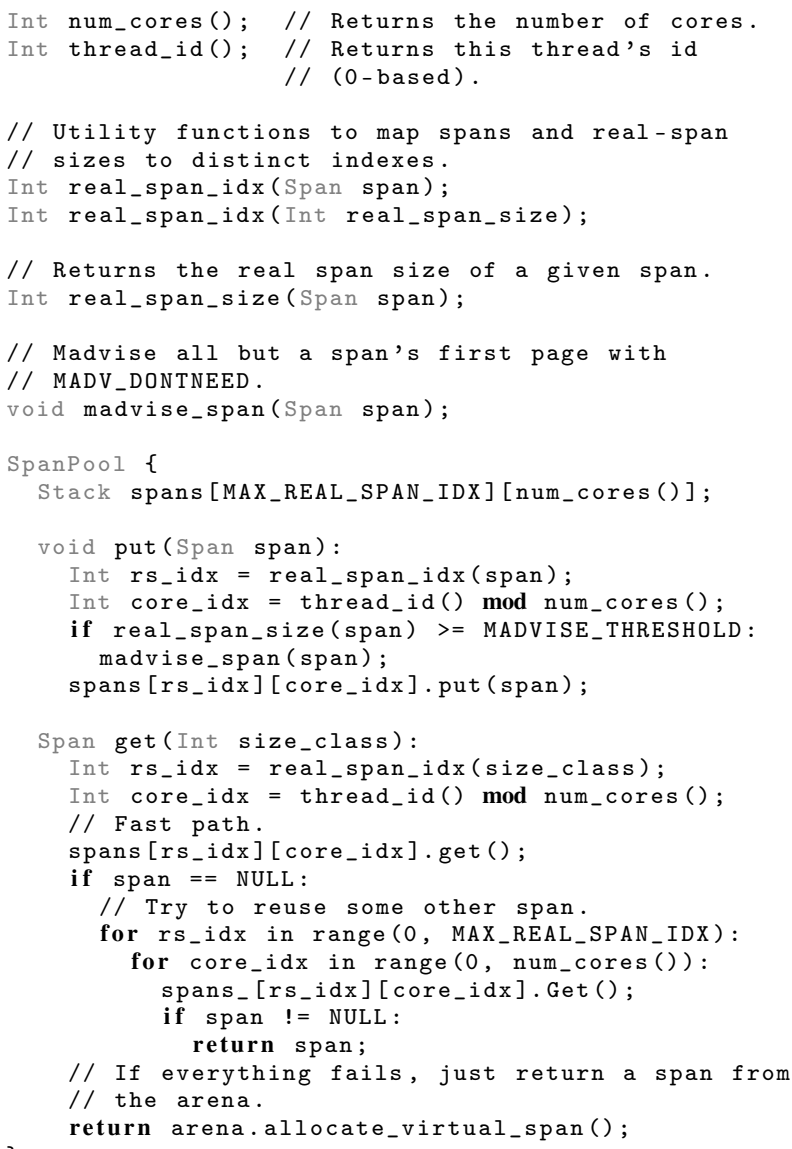

a smaller real-span size. The MADVISE_THRESHOLD (line 23) is set to $32 \mathrm{~KB}$, which is the boundary between real-span sizes of size classes that are incremented by 16 bytes and those that are incremented in powers of two. Note that lowering the threshold does not substantially improve the observed memory consumption in our experiments while it noticeably decreases performance. Furthermore, for scenarios where physical fragmentation is an issue, one can add a compaction call that traverses and madvises particular spans.

Upon retrieving a span from the span pool, for a given size class, a thread performing a get call first determines the real-span index of the size class (line 28) and the core index as thread identifier modulo the number of cores (line 29). In the fast path for span retrieval the thread then tries to retrieve a span from this identified stack (line 31). Note that this fast path implements the match to the put call, effectively maximizing locality for consecutively inserted) and retrieved spans of equal real-span sizes. If no span is found in the fast path, the thread searches all real-span size indexes and core indexes for a span to use (lines 34-38). Note that this motivates the design of the real-span sizes: For reuse, a span of a large real-span size has anyway been madvised whereas

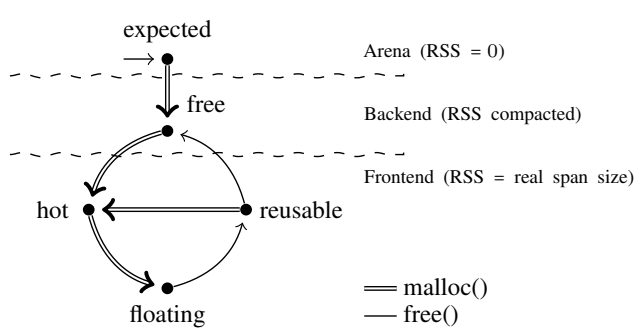

Figure 3: Life cycle of a span

all other spans have the same real-span size; Reusing a span in the same real-span size (even if the size class changes) amounts only to changing the header. Only when the search for an empty virtual span fails, the thread gets a new virtual span from the arena (as for initial allocation; line 41). Note that the search through the span-pool may fail even if there are spans in it due to the global use of the arrays (and the nonlinearizable emptiness check).

\subsection{Frontend: Allocation and Deallocation}

We now explain the mutator-facing frontend of scalloc, i.e., the part of the allocator that handles allocation and deallocation requests from the mutator.

We distinguish several states in which a span can be, illustrated in Figure 3. A span can be in a state: expected, free, hot, floating, or reusable. A span is expected if it is still in the arena, i.e., it is completely unused. Note that in this state its memory footprint is 0 bytes. Spans contained in the span-pool are free. A span can be in some of the other states only when it is in the frontend, i.e., it is assigned a specific size class. Spans that are hot are used for allocating new blocks. For spans that are not hot we distinguish between floating and reusable based on a threshold of the number of free blocks. Spans with less than or equal free blocks than the specified threshold are floating, spans with more free blocks than specified by the threshold are reusable. We refer to this threshold as reusability threshold. It is possible to only have spans that are floating, i.e., no reuse of nonempty spans, at the expense of increased fragmentation. Throughout its life in the frontend, a span is always assigned to exactly one local-allocation buffer (LAB), the so-called owning LAB. By default LABs are TLABs in scalloc, i.e., each LAB has a owner thread. Alternatively, scalloc can also be configured to use core-local allocation buffers (CLABs), i.e., one LAB per core where threads with equal identifiers modulo number of cores share the same LAB. Either way, in each LAB and for each size class there is a unique hot span. Furthermore, each LAB contains for each size class a set of reusable spans. More details about this set are given in Section 5.

A consequence of the concept of ownership is that deallocation of a block may happen in spans that are not owned by a thread. We refer to such deallocation as remote free, whereas deallocation in a span owned by a thread is a local 
free. All allocations in scalloc are done locally, performed in the corresponding hot span. A common problem in allocator design is handling remote frees in a scalable way. Having no mechanism for handling remote frees results in so-called blowup fragmentation [3], i.e., any memory freed through remote frees cannot be reused again. Similar to other spanbased allocators [23, 27], scalloc provides two free lists of blocks in each span, a local free list and a remote free list. The local free list is only accessed by an owning thread, while the remote free list can be accessed concurrently by multiple (not owning) threads at the same time.

Allocation. Upon allocation of a block in a given size class, a thread checks its LAB's size class for a hot span. If a hot span exists the thread tries to find a block in the local free list of the hot span. If a block is found, the thread allocates in this block (this is the allocation fast path). The following situations can also occur (for implementation details see Section 5):

(a) No hot span exists in the given size class. The thread then tries to assign a new hot span by trying to reuse one from the set of reusable spans. If no span is found there, the thread falls back to retrieving a span from the span-pool.

(b) There is a hot span, but its local free list is empty. The idea now is to use a remotely freed block. However, it is not a wise option to allocate in the remote free list, as that would make allocation interfere with remote frees, destroying the performance of allocation. Therefore, this is a point of choice: If there are enough blocks (in terms of the reusability threshold) in the remote free list, the thread moves them all to its local free list and continues with fast-path allocation. Otherwise, if there are not enough blocks in the remote free list, the thread gets a new hot span like in (a).

Deallocation. Upon deallocation, a thread returns the block to be freed to the corresponding free list, which is the local free list in case where the thread owns the span, and the remote free list otherwise. Depending on the state of the span where the block is allocated, the thread then performs the following actions (for implementation details see Section 5):

(a) The span is floating. If the number of free blocks in this span is now larger than the reusability threshold, the span's state changes to reusable and the span is inserted into the set of reusable spans of the owning LAB.

(b) The span is reusable. If the free was the last free in the span, i.e., all blocks have been freed, the span is removed from the set of reusable spans of the owning LAB and returned to the span-pool.

If the span is hot, no additional action is taken.

Note that a new contribution of scalloc is that a span is freed upon the deallocation of the last object in the span. All other span-based allocators postpone freeing of a span until the next allocation which triggers a cleanup.

\section{Properties}

Span-internal Fragmentation. Span-internal fragmentation is a global property and refers to memory assigned to a realspan of a given size class that is currently free (unused by the mutator) but cannot be reused for serving allocation requests in other size classes by any LAB.

Let $f$ be the current global span-internal fragmentation. Let $s$ refer to the span on which the next operation happens. Let size be the size class of $s$ and $u$ be the size of the payload (memory usable for blocks) of $s$. At initialization $f=0$.

Then, for an allocation of a block in $s$

$$
f= \begin{cases}f+u-s i z e & \text { if no usable span } \\ f-\text { size } & \text { otherwise }\end{cases}
$$

where no usable spans means no hot span and no reusable spans are present (1). Note that a span might already be reusable (with respect to the threshold) but not yet present in the set of reusable spans. This case is still covered by (1) and is a result of the fact that freeing an object and further processing it (reusable sets, or span-pool) are operations performed non-atomically.

Furthermore, for a deallocation of a block in $s$

$$
f= \begin{cases}f-u & \text { if last block } \\ f+\text { size } & \text { otherwise }\end{cases}
$$

where last block (3) refers to the last free of a block in a given span. Note that to achieve this fragmentation property on a free call an allocator, such as scalloc, has to return an empty span to a global backend immediately. A regular free not emptying the span increases fragmentation by the size of the block as this span cannot be reused globally (4).

Operation Complexity. An allocation operation only considers hot spans and reusable spans and does not need to clean up empty spans. The operation is constant-time as in the uncontended case either a hot span is present and can be used for allocating a block or a reusable span is made hot again before allocating a block in it. In the contended case more than one reusable span may need to be considered because of concurrent deallocation operations. At least one of the operations will make progress in constant time.

A deallocation operation only considers the affected span, i.e., the span containing the block that is freed. Local deallocations are constant-time and remote deallocations are constanttime modulo synchronization (insertion into the remote freelist which is lock-free). Spans that get reusable are made reusable in constant-time modulo synchronization (insertion into the set of reusable spans which is lock-based). Spans that get empty are handled in the span-pool.

Span-pool put and get operations are constant-time modulo synchronization (the span-pool is lock-free). 


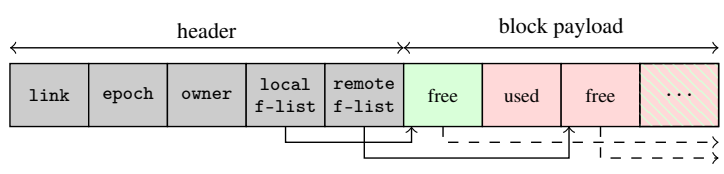

Figure 4: Real span layout

\section{Implementation Details}

We now explain the implementation details of scalloc, i.e., the encoding of fields in headers and the concrete algorithms used for allocation, deallocation, and thread termination.

The real-span header layout is shown in Figure 4. A link field is used to link up spans when necessary, i.e., it is used to link spans in the span-pool as well as in the set of reusable spans. The epoch field is used to uniquely identify a span's state within its life cycle (see below). The local and remote free list contained in a span are encoded in the fields local $f$-list and remote $f$-list, respectively. A span's owning LAB is encoded in the owner field.

The fields of a LAB are: an owner field that uniquely identifies a LAB; for each size class a field that refers to the hot span, called hot_span; and per size class the set of reusable spans kept in a field reusable_span.

Owner encoding. The owner field consists of two parts, an actual identifier (16 bits) of the owning LAB and a reference (48 bits) to the owning LAB. The whole field fits in a single word and can be updated atomically using compare-and-swap instructions. Note that upon termination of the last thread that is assigned to a LAB, the owner is set to TERMINATED. Subsequent reuses of the LAB (upon assigning newly created threads to it) result in a different owner, i.e., the actual identifier is different while the reference to the LAB stays the same. Also note that due to thread termination a span's owning LAB might have a different owner than the span's owner field indicates.

Epoch encoding. The epoch field is a single word that encodes a span's state and an ABA counter. The states hot, free, reusable, and floating are encoded in the upper parts (bitwise) of the word. The ABA counter (encoded in the rest of the word) is needed for versioning spans as the state alone is not enough to uniquely encode a state throughout a span's life cycle. E.g., one thread can observe a reusable span that after the last free is empty. Since freeing the object and transitioning the span into the state is not an atomic operation, another thread can now observe this span as empty (because it has been delayed after an earlier free operation) and put it into the span-pool. This span can now be reused by the same thread in the same size class ultimately ending up in the state reusable, but not completely empty. At this point the thread that initially freed the last block in the previous round needs to be prevented from transitioning it into state free.

Remote f-list encoding. We use a Treiber stack [29] to implement the remote free list in a span. The top pointer of the stack is stored in its own cache line in the span header.
Listing 2: Auxiliary structures and methods

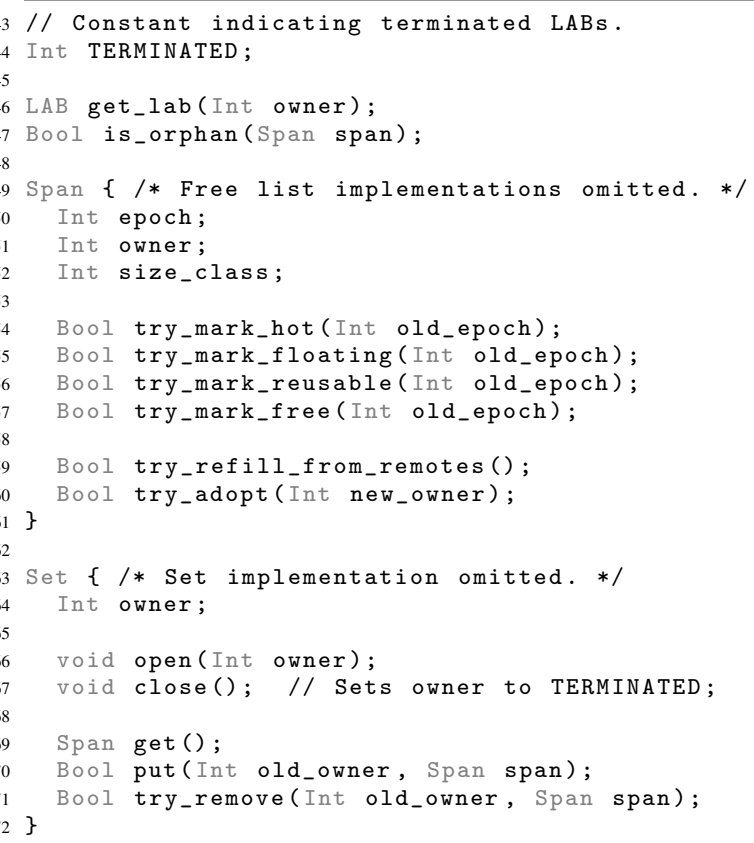

Furthermore, we keep the number of blocks in the stack's top pointer. This number is increased on each put operation. A single call is used to retrieve all blocks from this free list by atomically setting the stack's top pointer to NULL (implicitly setting the block count to 0 ). Note that generating a new state (putting and retrieving all blocks) only requires the top pointer. As a result special ABA handling is not needed (ABA can occur, but is not a problem) ${ }^{3}$.

Listing 2 provides an overview of auxiliary methods on spans and sets for reusable spans.

Recall that an owner field embeds a reference to the corresponding $\mathrm{LAB}$, which can be retrieved using get_lab (line 46). Furthermore, the function is_oprhan (line 47) is used to check whether the given span is an orphan, i.e., all threads assigned to its owning LAB have terminated before all blocks have been returned.

A span then contains the previously mentioned epoch and owner fields (lines 50-51). The methods that try to mark a span as being in a specific state (lines 54-57) all take an epoch value and try to atomically change it to a new value that has the corresponding state bits set and the ABA counter increased. These calls are then used in the actual algorithm for allocation and deallocation to transition a span from one state into another. The method try_refill_from_remotes (line 59) is used to move remote blocks (if there are more available then reusability threshold) from the remote free list to the local one. The

\footnotetext{
${ }^{3}$ For detailed explanations of the ABA problem see [24].
} 
method try_adopt (line 60) is used to adopt orphaned spans, i.e., atomically change their owning LAB.

Maintaining reusable spans should not have a noticeable performance impact (latency of allocation and deallocation) - which of course suggests using fast and scalable rather than non-scalable and slow data structures. Our design provides constant time put, get, and remove of arbitrary spans (lines 69-71). Furthermore, reusable spans are cleaned upon termination of the last thread assigned to a $\mathrm{LAB}$, requiring open and close methods (lines 66-67) that effectively prohibit put and remove methods accessing a set when no owner (i.e. TERMINATED) is present or the owner is different than the one provided as parameter. For details on thread termination see below. Contention on sets of reusable spans is low as the sets are segregated by size class and LABs. For the implementation of reusable sets of spans in scalloc we use a lock-based deque. We are aware of lock-free implementations of deques [7] that can be enhanced to be usable in scalloc. However, the process of cleaning up the set at thread termination (see below) requires wait-freedom as other threads may still be accessing the data structure. Helping approaches can be used to (even efficiently [19]) solve this problem. Experiments suggest that contention on these sets is low and we thus keep the implementation simple.

Listing 3 illustrates the main parts of scalloc's frontend. For simplicity we omit error handling, e.g. returning out of memory. Recall that each LAB is assigned an owner and holds for each size class a reference to the hot_span and the reusable_spans (lines 74-76).

The method get_span (line 80) is used to retrieve new spans, either from the reusable spans (lines 82-87), or from the span-pool (line 88). The calls to try_mark_hot on line 85 and line 89 represent the transitions free $\rightarrow$ hot and reusable $\rightarrow$ hot, respectively. Note that the transition free $\rightarrow$ hot does not compete with any other threads.

The method allocate (line 92) is used to allocate a single block in a hot span. If no hot span is present a new one is obtained using get_span (line 94). The hot span is then used to retrieve a block from the local free list of a span (line 95). If this attempt fails because the local free list is empty, the remote free list is inspected. If enough (with respect to reusability threshold represented as REUSABILITY_THRESHOLD) remotely freed blocks are available, they are moved to the local free list (line 98), just before actually allocating a new block (line 99). If not enough remotely freed blocks are available the current hot span is marked as floating (line 101), i.e., the hot span takes the transition hot $\rightarrow$ floating, and a new hot span is retrieved (line 102). The block is then allocated in the new hot span (line 103).

The method deallocate (line 106) is used to free a single block. Since freeing a block and transitioning spans through states are non-atomic operations, the owner and epoch values of a span are stored before freeing the
Listing 3: Frontend: Allocation, deallocation, and thread termination and initialization

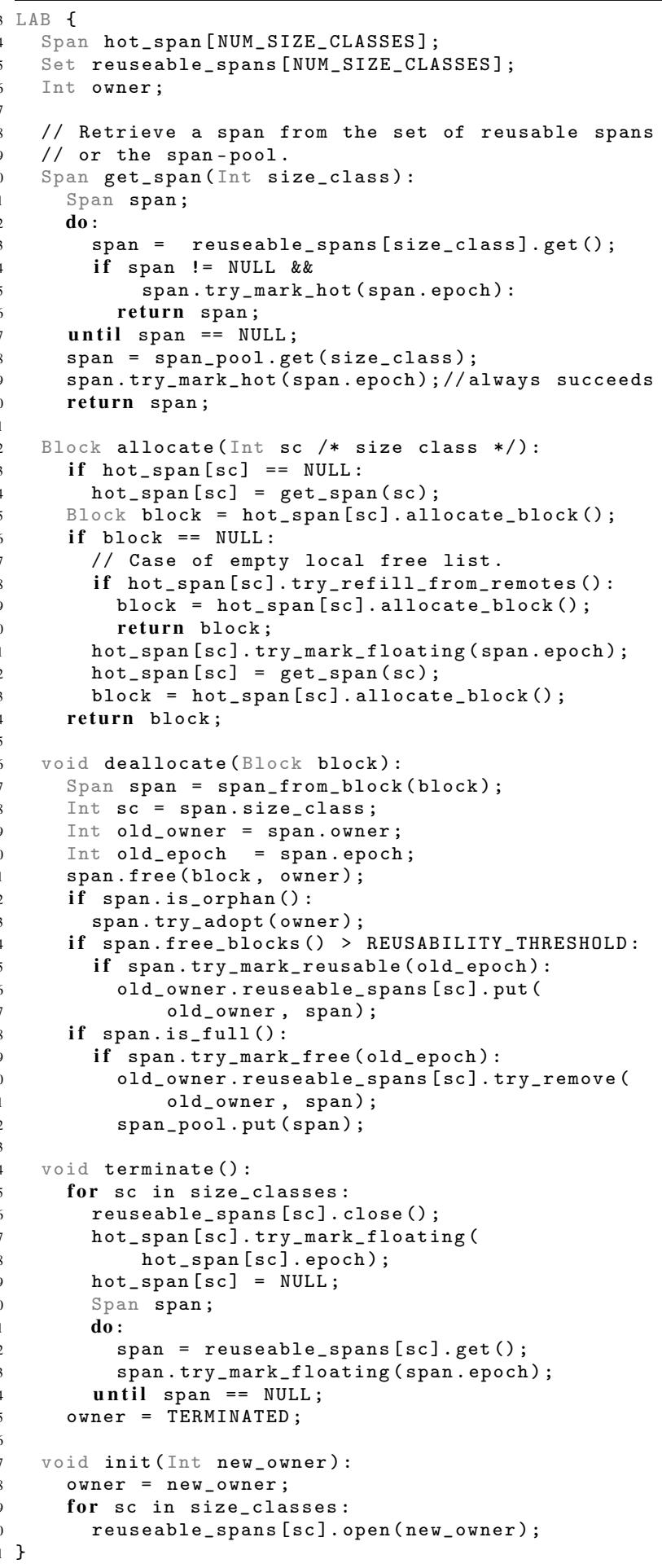

block (lines 109-110). The span's free call (line 111) then puts the block into the corresponding free list (local or remote, depending on the owner of the span). If after this free, the 
number of free blocks is larger than the reusability threshold (line 114), the span is put into state reusable (floating $\rightarrow$ reusable). Similar to other state transitions, this action is serialized through try_mark_reusable (line 115). The succeeding thread then also tries to insert the span into the set of reusable spans for this size class (line 117). Note that this call takes the old owner as parameter to prohibit inserting into a reusable set of a LAB that has either no owner or an owner that is different from the old owner. Similarly, making the transition reusable $\rightarrow$ free requires marking it as free through try_mark_free (line 119). Note that marking a span as free competes with reusing it in get_span. After successfully marking it as free the span can be removed (if needed) from the set of reusable spans (line 121). Finally, the span is put into the span-pool (line 122).

Thread Termination. Similar to others [17], we refer to spans that have not yet been transitioned into the state free (because they contain live blocks) while all threads assigned to their owning LAB have terminated as orphaned spans. Since LABs do not necessarily have references to all owned spans (there exist no references to floating spans) a span cannot be declared as orphaned by setting a flag. Instead, orphaned spans can be detected by comparing a span's owner against the owner that is set in the owning LAB. Owner fields that differ or a LAB owner set to TERMINATED indicates an orphaned span. Orphaned spans are always floating and adopted by threads upon freeing a block in these spans (lines 112-113). LAB cleanup happens in terminate (line 124) where for each size class (lines 125-134) the reusable spans set is closed (line 126), and all spans (hot and reusable) are transitioned into state floating (line 128 and line 133). For reusable spans this transition competes with reusable $\rightarrow$ free (line 119), where a potential last free of a block in a span triggers putting the span into the span-pool. Finally, the LAB is marked as terminated and consequently all spans that are not free can be observed as orphaned. Reusing a LAB later on requires setting a new unique owner (line 140).

Handling Large Objects. Scalloc provides span-based allocation of blocks of size less than or equal to $1 \mathrm{MB}$ and relies on conventional mmap for all other objects. For allocation this means that the frontend just forwards the allocation request to an allocator that just mmaps the block and adds a header containing the required size. Deallocation requires checking whether a block has been allocated in a span or not. However, since spans are contained in a single large arena this check is cheap (xor-ing against aligned arena boundary). Depending on whether the block has been allocated in a span or not, the request is just forwarded appropriately.

Unwritten Rules. The illustrated concepts yield a design that provides scalability on a multi-core system while keeping memory compact with respect to to a reusability threshold. To this end we would like to note that being competitive in absolute terms requires an implementation that forces strict inlining of code, careful layout of thread-local storage, and intercepting thread creation and termination. Without those techniques absolute performance suffers from overheads of function calls as well as cache misses (for unnecessarily checking conditions related to thread-local storage).

\section{Related Work}

We first discuss related work on concurrent data structures and then on existing concurrent memory allocators.

Concurrent data structures in the fast path of the frontend as well as the backend of scalloc are lock-free [16]. A recent trend towards semantically relaxed concurrent data structures [1, 15] opens doors for new design ideas and even greater performance and scalability so that hierarchies of spans (buffers in general) can be avoided and may be utilized globally across the whole system. The concurrent data structures in scalloc are pools, allowing in principle every data structure with pool semantics to be used. However, unlike segment queues [1] and $k$-Stacks [15], Distributed Queues [11] with Treiber stacks [29], as used in scalloc, do not require dynamically allocated administrative memory (such as sentinel nodes), which is important for building efficient memory allocators. The data structures for reusable spans within a TLAB are implemented using locks but could in principle be replaced with wait-free sets, which nowadays can be implemented almost as efficiently as their lock-free counterparts [19].

Many concepts underlying scalloc such as size classes, hierarchical allocation (local allocation buffers, spans), and local memory caching in so-called private heaps (span ownership) have already been introduced and studied in thread-local variants $[3,30]$. Scalloc borrows from some of these concepts and integrates them with lock-free concurrent data structures, and introduces new ideas like virtual spans.

In our experiments we compare scalloc to some of the best and most popular concurrent memory allocators: Hoard (git-13c7e75), jemalloc (3.6.0), llalloc (1.4), ptmalloc2 ${ }^{4}$ (libc 2.19), Streamflow (git-41aa80d), SuperMalloc (git-bd7096f), Intel TBB allocator (4.3), and TCMalloc (googleperftools 2.1). McRT-Malloc [17] is left out of our comparison because of a missing implementation. For Michael's allocator [25] there exists no reference implementation - an implementation for x86-64 by the Streamflow authors crashes for all our benchmarks; we have received another implementation ${ }^{5}$ which unfortunately does not perform and scale as we expect from the original paper. We thus decided to leave the Michael allocator out of our comparisons. ptmalloc2 [9] extends Doug Lea's malloc [22] (dlmalloc; 2.7.x) and is part of the GNU libc library. jemalloc [8] is the default allocator in FreeBSD and NetBSD and has been integrated into prod-

\footnotetext{
${ }^{4}$ Since ptmalloc3 performs worse than ptmalloc2, we exclude ptmalloc3 from our experimental evaluation.

${ }^{5}$ In a private correspondence we received pointers to the Amino Concurrent Building Blocks (http://amino-cbbs . sourceforge .net/) malloc implementation.
} 
ucts of Mozilla (like Firefox) and Facebook. TCMalloc [10] is Google's counter-part to jemalloc, also aiming at performance and scalability. TBB [18] is maintained by Intel as part of their thread building block library which aims at easy creation of fast and scalable multi-threaded programs. llalloc [23] is an allocator developed by Lockless Inc. Hoard [3] and Streamflow [27] are both academic allocators known to perform well. SuperMalloc [20] is another recently developed academic allocator introducing, to our knowledge independently, after scalloc's virtual spans, the idea of segmenting virtual address space for uniform treatment of different-sized objects. Note that the concept of virtual spans may readily be used in other allocators and is orthogonal to the actual allocator design. Moreover, mapping virtual memory in a scalable fashion, e.g. as in RadixVM [6], does not solve the problem of designing a competitive allocator. Memory fragmentation and system-call overhead still need to be managed.

All mentioned allocators create private heaps in one way or another. This design has proven to reduce contention and (partially) avoid false sharing. Scalloc is no different in this aspect as it also creates private heaps (span ownership) and exchanges space between threads (through the span-pool).

Another aspect all allocators have in common are heaps segregated by size classes for spatial locality. It makes object headers obsolete (except for coalescing which is why ptmalloc2 uses them).

Allocators implementing private heaps without returning remotely freed objects to the allocating threads suffer from unbounded blowup fragmentation in producer-consumer workloads [3]. Hence, it is necessary to transfer remotely freed memory back to the heap it was allocated on.

ptmalloc2 solves the blowup fragmentation problem by globally locking and then deallocating the block where it has been allocated. TCMalloc and jemalloc both maintain caches of remotely freed objects which are only returned to a global heap when reaching certain thresholds. Hoard allocates objects in superblocks which are similar to the spans in scalloc. Unlike scalloc, Hoard returns remotely freed objects in a hierarchical fashion, first by deallocating the objects in the superblocks in which they were allocated, then by transferring superblocks between private heaps, and eventually by transferring superblocks from private heaps to a global heap. For this purpose, Hoard locks the involved superblocks, private heaps, and the global heap. llalloc, Streamflow, and TBB maintain a private and a public free-list per thread and size class. The public free lists are implemented using lock-free algorithms. Scalloc does exactly the same.

To this end we would like to note that the frontends of llalloc and Streamflow are to some extent similar to scalloc's frontend. However, both allocators require cleaning up empty spans in allocation calls, and use different strategies for large objects and backends.

Another common practice in many allocators is to handle small and big objects, whatever the threshold between small and big is, in separate sub-allocators which are typically based on entirely different data structures and algorithms. jemalloc, llalloc, ptmalloc2, and TCMalloc are such hybrid allocators, whereas Hoard, scalloc, Streamflow, SuperMalloc, and TBB are not. ptmalloc2 manages big objects in best-fit free-lists, jemalloc and TCMalloc round the size of big objects up to page multiples and allocate them from the global heap, and llalloc maintains a binary tree of big objects (c.f. binary buddy system) in a large portion of memory obtained from the operating system. Huge objects, again whatever the threshold between big and huge is, are handled by the operating system in all considered allocators including scalloc. The principle challenge is to determine the thresholds between small and big, for hybrid allocators, as well as between big and huge, for all allocators. Scalloc addresses that challenge, through virtual spans, by removing the threshold between small and big objects and by making the threshold between big and huge so large that it is likely to be irrelevant in the foreseeable future for most existing applications.

A concept related to virtual spans called spaces appears in the Memory Management Toolkit (MMTk) for managed languages [5]. We note that the generally poor performance of SuperMalloc for concurrent workloads shows that virtual spans alone do not suffice for achieving competitive temporal and spatial performance and scalability. Virtual spans alone only simplify allocator design as they enable uniform treatment of small and big objects, and reduce memory consumption. As shown in our experimental evaluation, the combination of virtual spans with a scalable backend and a highperformance frontend is crucial for achieving competitive performance and scalability.

\section{Experimental Evaluation}

In our experiments we compare scalloc with other allocators and with other scalloc configurations on synthetic and real workloads. Our evaluation is structured in two parts. The first part of the evaluation covers well known allocator workloads from the literature (threadtest, shbench, larson) [3, 21, 26] as well as the single-threaded allocation intensive workload 483.xalancbmk from the SPEC CPU2006 suite [14] that are known to perform interesting usage patterns, e.g. threadtest provides a completely thread-local workload for batched allocation and deallocation of objects. Furthermore, the evaluation also employs workloads generated with ACDC [2], a benchmarking tool that can be configured to emulate virtually any relevant workload characteristic not covered by existing benchmarks, e.g. producer-consumer patterns, varying object sizes, and different object access patterns. The second part of the evaluation focuses on our design decisions implemented in scalloc. In particular, we show the impact of effectively disabling key features of scalloc like virtual spans.

We have also experimented with application benchmarks and generally found that scalloc either performs as other competitive allocators or better. However, in our and others' [25] 
experience most concurrent applications either use custom allocation or tailor their behavior so that very few threads allocate concurrently, as is the case in e.g. the Chrome web browser. We see our work as a step towards providing an infrastructure that changes this practice.

A summary of all benchmarks can be found in Table 1. A detailed description of each experiment is presented in the corresponding subsection.

All experiments ran on a UMA machine with four 10core $2 \mathrm{GHz}$ Intel Xeon E7-4850 processors supporting two hardware threads per core, $32 \mathrm{~KB} 8$-way associative L1 data cache and $32 \mathrm{~KB}$ 4-way associative L1 instruction cache per core, $256 \mathrm{~KB}$ unified 8-way associative L2 cache per core, 24MB unified 24-way associative L3 cache per processor, 128GB of main memory, and Linux kernel version 3.8.0.

Note that recent Linux kernels provide the ability to use transparent huge pages ${ }^{6}$ as backing store for regular pages, i.e., huge pages can be used by the kernel as physical page frames for regular pages. Since this feature interferes with any mechanism relying on on-demand paging, e.g. calling madvise to return memory, we have disabled it in all experiments except one where we use it to disable virtual spans intentionally. Transparent huge pages also impact other allocators, e.g. jemalloc.

There are two configurable parameters in scalloc, MADVISE_THRESHOLD and REUSABILITY_THRESHOLD. We choose MADVISE_THRESHOLD to be $32 \mathrm{~KB}$ which is the smallest real-span size. In principle one can set the threshold as low as the system page size, effectively trading performance for lower memory consumption, but as spans are subject to reuse at all times this is not necessary. Furthermore we choose REUSABILITY_THRESHOLD to be $80 \%$, i.e., spans may be reused as soon as $80 \%$ of their blocks are free. Span reuse is useful in workloads that exhibit irregular allocation and deallocation patterns. Since span reuse optimizes memory consumption with negligible overhead we have also considered a configuration that does not reuse spans and discuss the results but do not show the data for clarity.

Unless explicitly stated we report the arithmetic mean of 10 sample runs including the $95 \%$ confidence interval based on the corrected sample standard deviation. For memory consumption we always report the resident set size (RSS). The sampling frequency varies among experiments and has been chosen high enough to not miss peaks in memory consumption between samples. Since most benchmarks do not report memory consumption we employ an additional process for measuring the RSS. As a result, benchmarks like threadtest, larson, and shbench only scale until 39 threads. We still report the 40 threads ticks to illustrate this behavior.

\footnotetext{
${ }^{6}$ See https://www.kernel.org/doc/Documentation/vm/ transhuge.txt
}

Table 1: Summary of benchmarks

\begin{tabular}{|c|c|c|c|c|}
\hline BENCHMARK & $\begin{array}{c}\text { OBJECt SizE } \\
\text { [BYTES] }\end{array}$ & $\begin{array}{c}\text { LOCAL } \\
\text { FREES }\end{array}$ & $\begin{array}{c}\text { REMOTE } \\
\text { FREES }\end{array}$ & $\begin{array}{c}\text { THREAD } \\
\text { TERM. }\end{array}$ \\
\hline $\begin{array}{l}\text { SINGLE-THREA } \\
\text { 483.xalancbmk }\end{array}$ & ED $1-2 \mathrm{M}$ & $100 \%$ & $0 \%$ & no \\
\hline \multicolumn{5}{|c|}{ MuLTI-THREADED } \\
\hline Threadtest & 64 & $100 \%$ & $0 \%$ & no \\
\hline Shbench & $1-8$ & $100 \%$ & $0 \%$ & no \\
\hline Larson & $7-8$ & $\geq 99 \%$ & $<1 \%$ & yes \\
\hline Prod.-Cons. & $16-512$ & \multicolumn{2}{|c|}{ see Section 7.3} & no \\
\hline False Sharing & 8 & \multicolumn{2}{|c|}{ see Section 7.4} & no \\
\hline Object Sizes & $16-4 \mathrm{M}$ & $100 \%$ & $0 \%$ & no \\
\hline Spatial Locality & $16-32$ & $100 \%$ & $0 \%$ & no \\
\hline \multicolumn{5}{|c|}{ DESIGN DECISIONS } \\
\hline Virtual Spans & $16-4 \mathrm{M}$ & $100 \%$ & $0 \%$ & no \\
\hline Span-Pool & 256 & $100 \%$ & $0 \%$ & no \\
\hline Frontend & 64 & $100 \%$ & $0 \%$ & no \\
\hline
\end{tabular}

\subsection{Single-Threaded Workload: 483.xalancbmk}

We compare different allocators on the 483.xalancbmk workload of the SPEC CPU2006 suite which is known to be an allocation intense single-threaded workload [28].

Figure 5 reports a benchmark score called ratio where a higher ratio means a lower benchmark running time. The results omit data for Streamflow because it crashes. Scalloc (among others) provides a significant improvement compared to ptmalloc 2 but the differences among the best performing allocators including scalloc are small. Nevertheless, the results demonstrate competitive single-threaded temporal performance of scalloc. Note that the SPEC suite does not provide metrics for memory consumption.

\subsection{Thread-Local Workloads}

We evaluate the performance of allocators in workloads that only consist of thread-local allocations and deallocations. Recall that scalloc only allocates blocks in a hot span of a given size class. Hence, workloads that perform more consecutive allocations in a single size class than a span can hold blocks (i.e. the working set is larger than the real-span size) result in benchmarking the span-pool.

\subsubsection{Threadtest}

Threadtest [3] allocates and deallocates objects of the same size in a configurable number of threads and may perform a configurable amount of work in between allocations and deallocations. For a variable number of threads $t$, the benchmark is configured to perform $10^{4}$ rounds per thread of allocating and deallocating $\frac{10^{5}}{t}$ objects of 64 bytes. For temporal performance we show the speedup with respect to single-threaded ptmalloc2 performance.

In scalloc, objects of 64 bytes are allocated in 64-byte blocks in spans with a real-span size of $32 \mathrm{~KB}$. Since allocations and deallocations are performed in rounds reusing of 


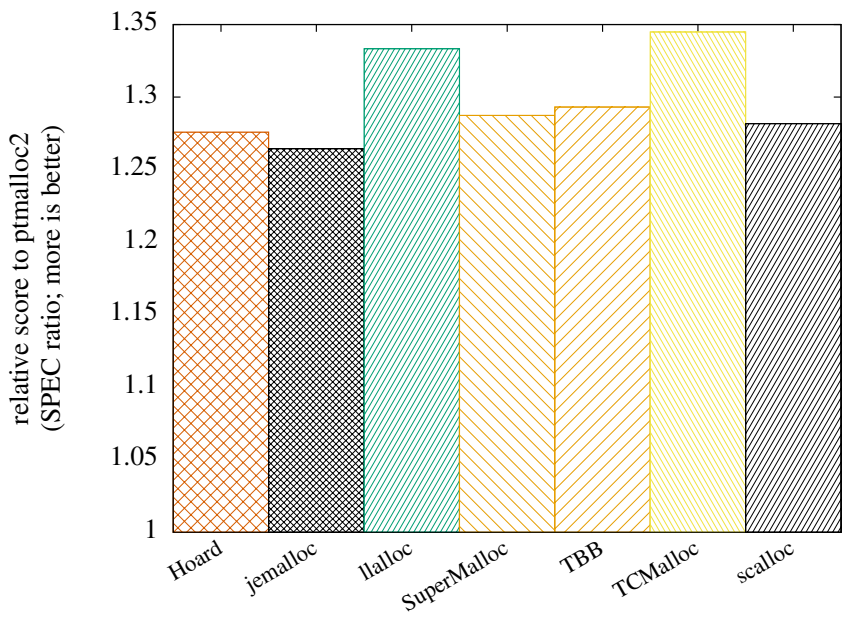

Figure 5: Single-threaded temporal performance: SPEC CPU2006 483.xalancbmk

spans has no effect on memory consumption. The overhead of adding and removing spans to the reusable set is negligible.

Figure 6a illustrates temporal performance where all allocators but jemalloc scale until 39 threads with only the absolute performance being different for most allocators. Since the working set for a single round of a thread is roughly 6.4MB, allocators are forced to interact with their backend. The results suggest that the span-pool with its strategy of distributing contention provides the fastest backend of all considered allocators. The memory consumption shown in Figure $6 \mathrm{~b}$ suggests that threads do not exhibit a lock-step behavior, i.e., they run out of sync with respect to their local rounds, which ultimately manifests in lower memory consumption for a larger number of threads. The span-pool supports this behavior by providing a local fast path with a fall back to scalable global sharing of spans.

\subsubsection{Shbench}

Similar to threadtest, shbench [26] exhibits a thread-local behavior where objects are allocated and deallocated in rounds. Unlike threadtest though, the lifetime of objects is not exactly one round but varies throughout the benchmark. For a variable number of threads $t$, the benchmark is configured to perform $10^{6}$ rounds per thread of objects between 1 and 8 bytes in size. We exclude Streamflow from this experiment because it crashes for more than 1 thread in the shbench workload. For temporal performance we show the speedup with respect to single-threaded ptmalloc2.

Figure $7 \mathrm{a}$ shows the performance results. As objects survive rounds of allocations the contention on the span-pool is not as high as in threadtest. The memory consumption in Figure $7 \mathrm{~b}$ indicates that the absolute performance is determined by span sizes (or other local buffers). Allocators that keep memory compact in this benchmark also suffer from degrading absolute performance. Scalloc performs better than all other allocators except for llalloc which consumes more memory. Note that reusing spans in this benchmark has an impact on memory consumption. Scalloc is configured to reuse at $80 \%$ free blocks in a span. Disabling reusing of spans results for 20 threads in a memory consumption increase of $14.7 \%$ (while having no noticeable impact on performance). This suggests that reusing spans in scalloc is important in non-cyclic workloads. Note that the decrease of memory consumption at 6 threads is a workload artifact. In contrast, the peak in memory consumption for llalloc at 20 threads (which is repeatable) suggests an allocator artifact.

\subsubsection{Larson (Thread Termination)}

The larson benchmark [21] simulates a multi-threaded server application responding to client requests. A thread in larson receives a set of objects, randomly performs deallocations and allocations on this set for a given number of rounds, then passes the set of objects on to the next thread, and finally terminates. The benchmark captures robustness of allocators for unusual allocation patterns including terminating threads. Unlike results reported elsewhere [27] we do not observe a ratio of $100 \%$ remote deallocations as larson also exhibits thread-local allocation and deallocation in rounds. For a variable number of threads $t$, the benchmark is configured to last 10 seconds, for objects of 7 bytes (smallest size class for all allocators), with $10^{3}$ objects per thread, and $10^{4}$ rounds per slot of memory per thread. Unlike the other experiments, the larson benchmark runs for a fixed amount of time and provides a throughput metric of memory management operations per second. We exclude Streamflow from this experiment as it crashes under the larson workload.

Figure 8a illustrates temporal performance where all considered allocators scale but provide different speedups. Similar to shbench, the rate at which spans get empty varies. The memory consumption in Figure 8b illustrates that better absolute performance comes at the expense of increased memory consumption. Furthermore, terminating threads impose the challenge of reassigning spans in scalloc (and likely also impose a challenge in other allocators that rely on thread-local data structures). Similar to shbench, reusing spans before they get empty results in better memory consumption. Disabling span reuse increases memory consumption by $7.6 \%$ at 20 threads while reducing performance by $2.7 \%$.

\subsection{Producer-Consumer Workload}

This experiment evaluates the temporal and spatial performance of a producer-consumer workload to study the cost of remote frees and possible blowup fragmentation for an increasing number of producers and consumers. For that purpose we configure ACDC such that each thread shares all allocated objects with all other threads, accesses all local and shared objects, and eventually the last (arbitrary) thread accessing an object frees it. The probability of a remote free in the presence of $n$ threads is therefore $1-1 / n$, e.g. running 


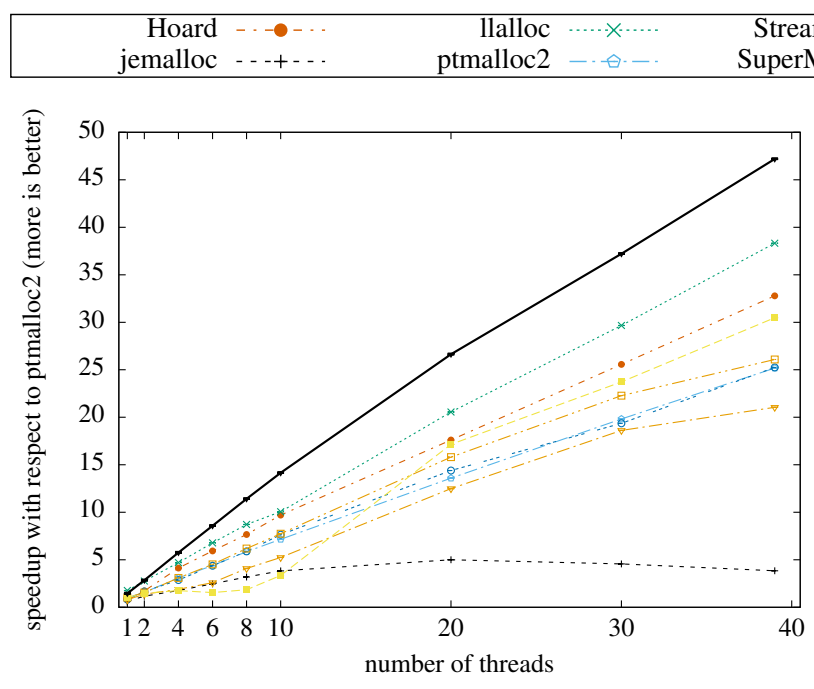

(a) Speedup

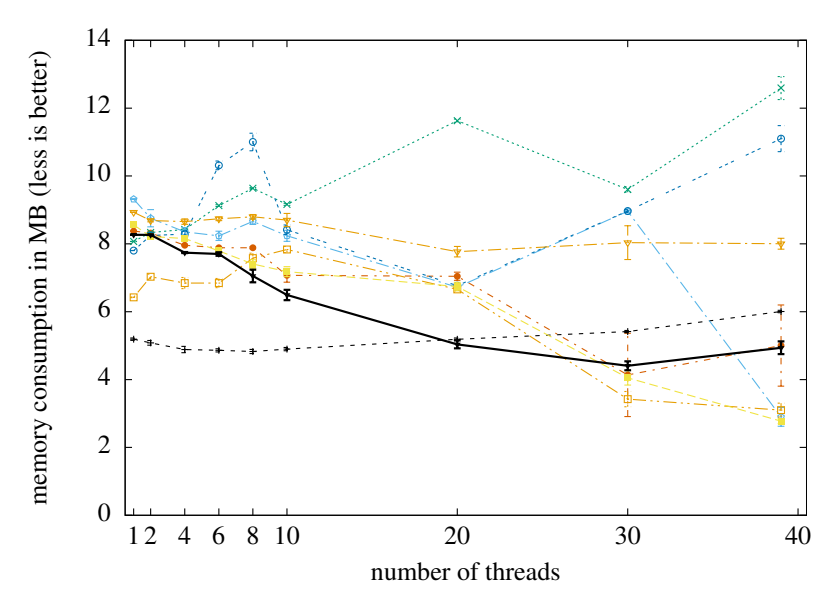

(b) Memory consumption

Figure 6: Thread-local workload: Threadtest benchmark

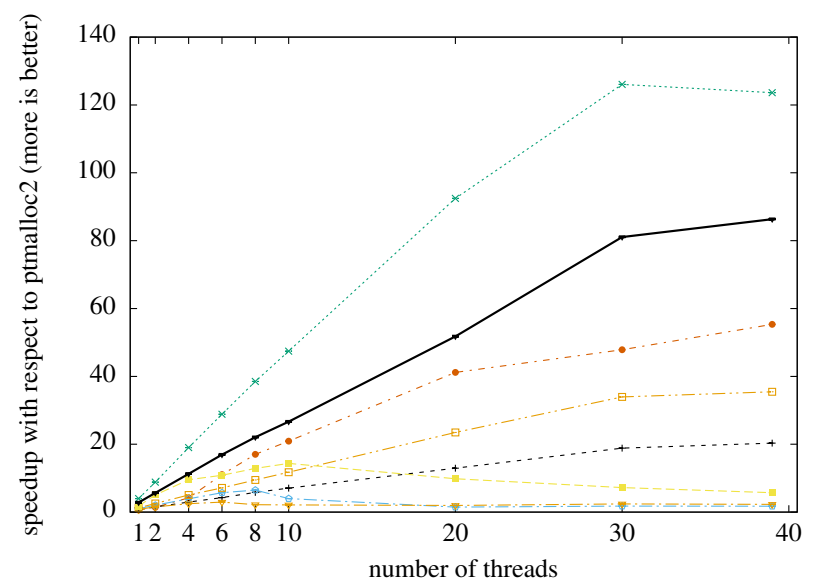

(a) Speedup

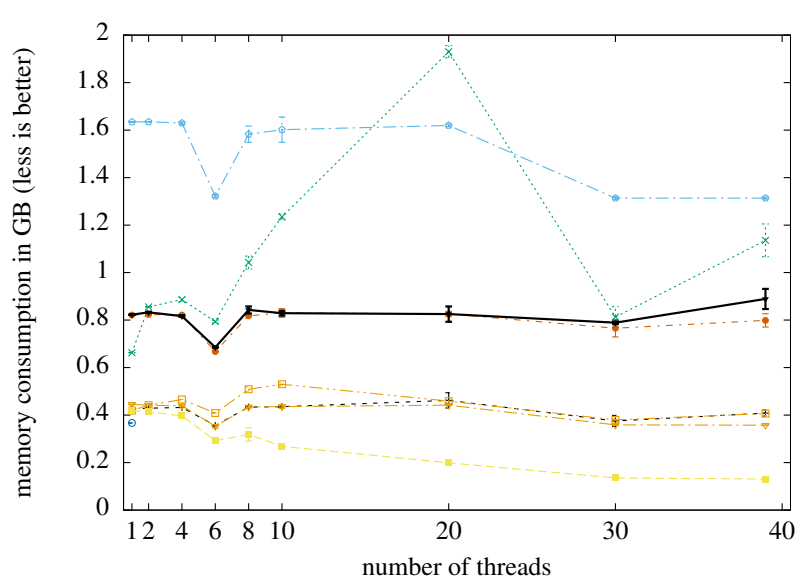

(b) Memory consumption

Figure 7: Thread-local workload: Shbench benchmark

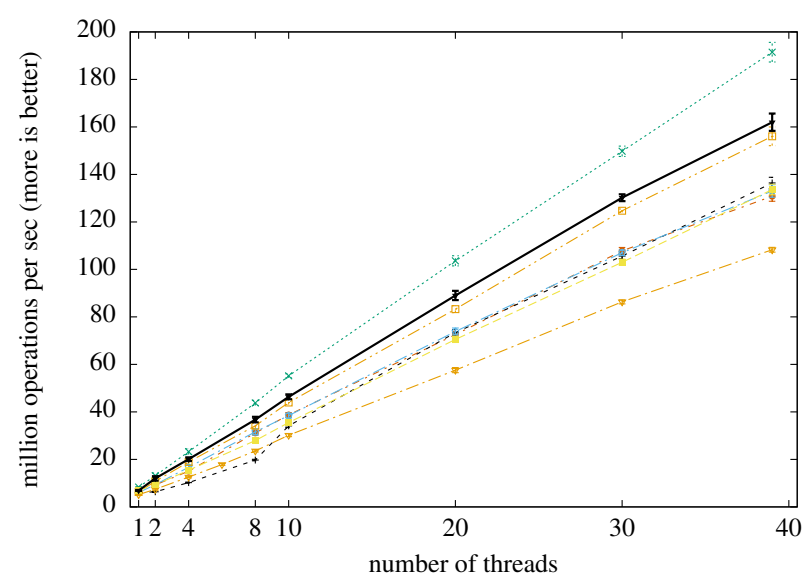

(a) Throughput

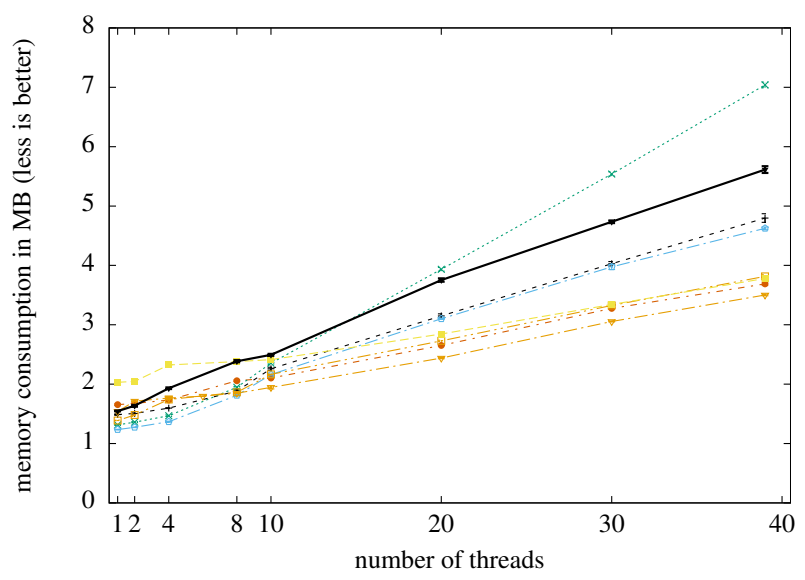

(b) Memory consumption

Figure 8: Thread-local workload (including thread termination): Larson benchmark 


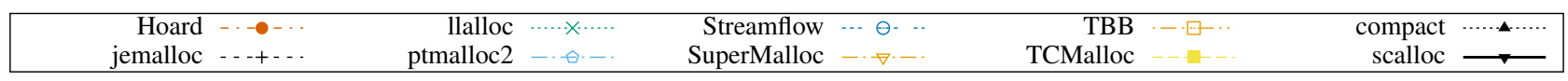

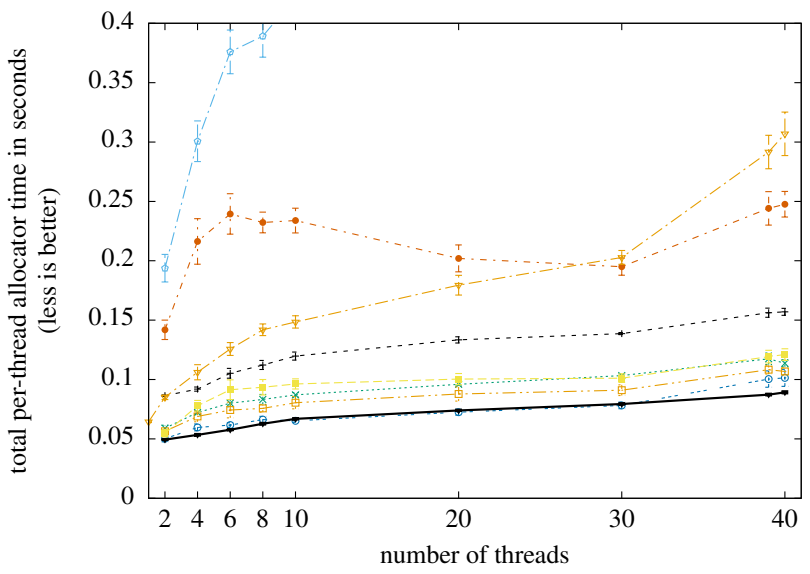

(a) Total per-thread allocator time

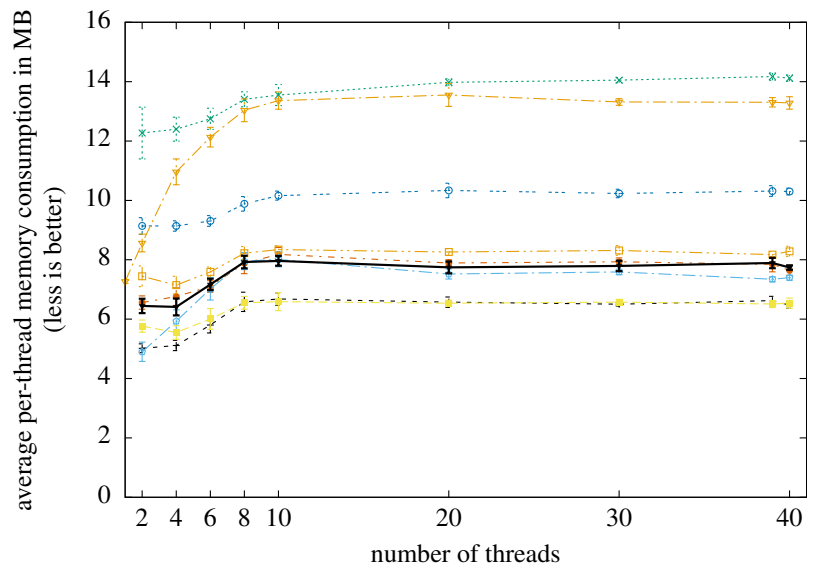

(b) Average per-thread memory consumption

Figure 9: Temporal and spatial performance for the producer-consumer experiment

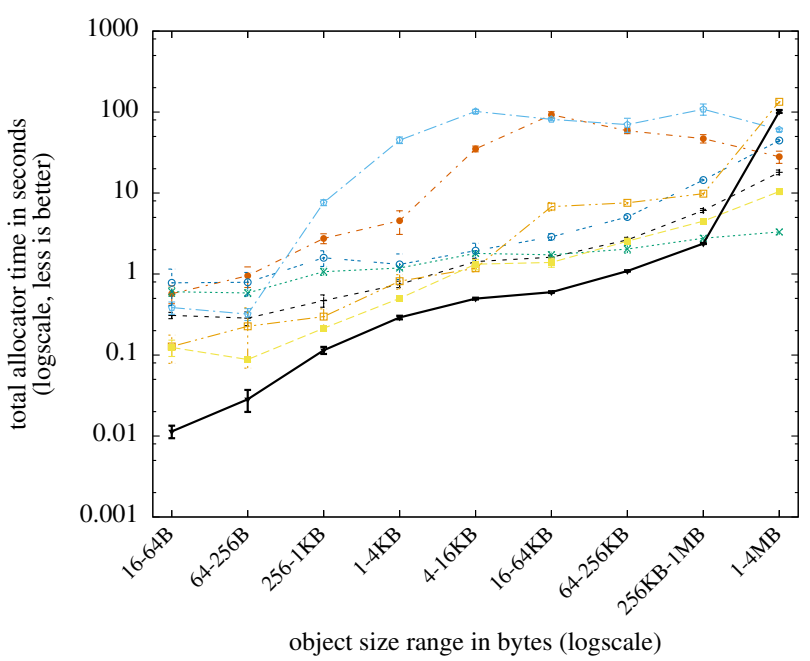

(a) Total allocator time

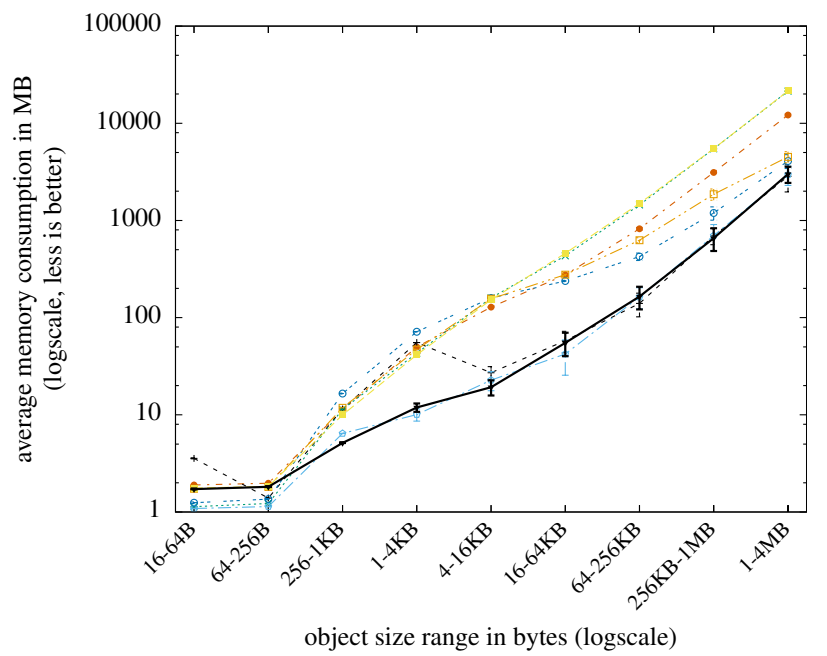

(b) Average memory consumption

Figure 10: Temporal and spatial performance for the object-size robustness experiment at 40 threads

two threads causes on average 50\% remote frees and running 40 threads causes on average $97.5 \%$ remote frees.

Figure 9a presents the total time each thread spends in the allocator for an increasing number of producers/consumers. Up to 30 threads scalloc and Streamflow provide the best temporal performance and for more than 30 threads scalloc outperforms all other allocators.

The average per-thread memory consumption illustrated in Figure 9b suggests that all allocators deal with blowup fragmentation, i.e., we do not observe unbounded growth in memory consumption. However, the absolute differences among different allocators are significant. Scalloc provides competitive spatial performance where only jemalloc and ptmalloc2 require less memory at the expense of higher total per-thread allocator time.
This experiment demonstrates that the approach of scalloc to distributing contention across spans with one remote free list per span works well in a producer-consumer workload and that using a lock-based implementation for reusing spans is not a performance bottleneck.

\subsection{Robustness against False Sharing}

False sharing occurs when objects that are allocated in the same cache line are read from and written to by different threads. In cache coherent systems this scenario can lead to performance degradation as all caches need to be kept consistent. An allocator is prone to active false sharing [3] if objects that are allocated by different threads (without communication) end up in the same cache line. It is prone to passive false sharing [3] if objects that are remotely 
deallocated by a thread are immediately usable for allocation by this thread again.

We have conducted the false sharing avoidance evaluation benchmark from Berger et. al. [3] (including active-false and passive-false benchmarks) to validate scalloc's design. The results we have obtained suggest that most allocators avoid active and passive false sharing. However, SuperMalloc and TCMalloc suffer from both active and passive false sharing, whereas Hoard is prone to passive false sharing only. We omit the graphs because they only show binary results (either false sharing occurs or not). Scalloc's design ensures that in the cases covered by the active-false and passive-false benchmarks no false sharing appears, as spans need to be freed to be reused by other threads for allocation. Only in case of thread termination (not covered by the active-false and passive-false benchmarks) threads may adopt spans in which other threads still have blocks, potentially causing false sharing. We have not encountered false sharing with scalloc in any of our experiments.

\subsection{Robustness for Varying Object Sizes}

We configure the ACDC allocator benchmark [2] to allocate, access, and deallocate increasingly larger thread-local objects in 40 threads (number of native cores) to study the scalability of virtual spans and the span pool.

Figure 10a shows the total time spent in the allocator, i.e., the time spent in malloc and free. The $\mathrm{x}$-axis refers to intervals $\left[2^{x}, 2^{x+2}\right)$ of object sizes in bytes with $4 \leq x \leq 20$ at increments of two. For each object size interval ACDC allocates $2^{x} \mathrm{~KB}$ of new objects, accesses the objects, and then deallocates previously allocated objects. This cycle is repeated 30 times. For object sizes smaller than 1MB scalloc outperforms all other allocators because virtual spans enable scalloc to rely on efficient size-class allocation. The only possible bottleneck in this case is accessing the span-pool. However, even in the presence of 40 threads we do not observe contention on the span-pool. For objects larger than $1 \mathrm{MB}$ scalloc relies on mmap which adds system call latency to allocation and deallocation operations and is also known to be a scalability bottleneck [6].

The average memory consumption (illustrated in Figure 10b) of scalloc allocating small objects is higher (yet still competitive) because the real-spans for size-classes smaller than $32 \mathrm{~KB}$ have the same size and madvise is not enabled for them. For larger object sizes scalloc causes the smallest memory overhead comparable to jemalloc and ptmalloc2.

This experiment demonstrates the advantages of trading virtual address space fragmentation for high throughput and low physical memory fragmentation.

\subsection{Spatial Locality}

In order to expose differences in spatial locality, we configure ACDC to access allocated objects (between 16 and 32 bytes) increasingly in allocation order (rather than out-of-allocation order). For this purpose, ACDC organizes allocated objects

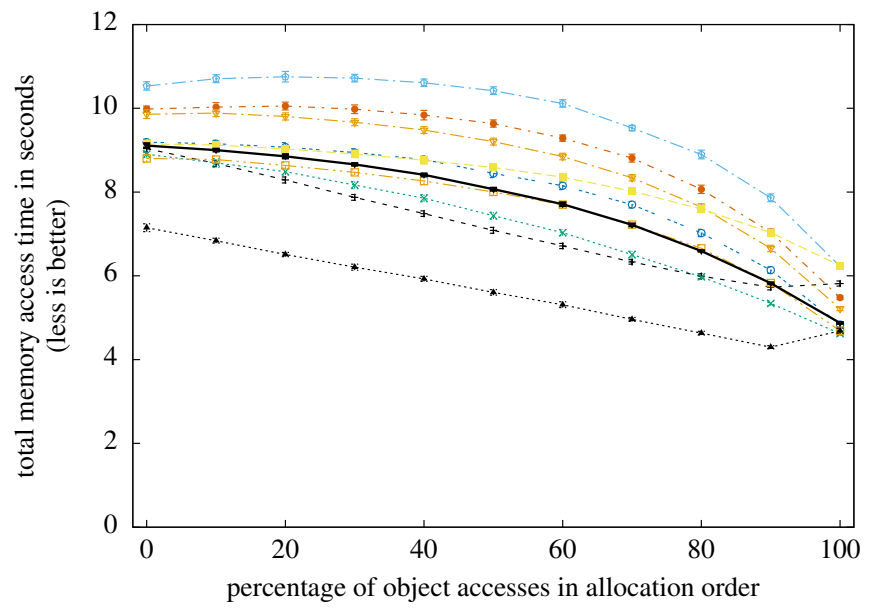

Figure 11: Memory access time for the locality experiment

either in trees (in depth-first, left-to-right order, representing out-of-allocation order) or in lists (representing allocation order). ACDC then accesses the objects from the tree in depth-first, right-to-left order and from the list in FIFO order. We measure the total memory access time for an increasing ratio of lists, starting at $0 \%$ (only trees), going up to $100 \%$ (only lists), as an indicator of spatial locality. ACDC provides a simple mutator-aware allocator called compact to serve as optimal (yet without further knowledge of mutator behavior unreachable) baseline. Compact stores the lists and trees of allocated objects without space overhead in contiguous memory for optimal locality.

Figure 11 shows the total memory access time for an increasing ratio of object accesses in allocation order. Only jemalloc and llalloc provide a memory layout that can be accessed slightly faster than the memory layout provided by scalloc. Note that scalloc does not require object headers and reinitializes span free-lists upon retrieval from the spanpool. For a larger ratio of object accesses in allocation order, the other allocators improve as well but not as much as llalloc, scalloc, Streamflow, and TBB which approach the memory access performance of the compact baseline allocator. Note also that we can improve memory access time with scalloc even more by setting its reusability threshold to $100 \%$. In this case spans are only reused once they get completely empty and reinitialized through the span-pool at the expense of higher memory consumption. We omit this data for consistency reasons.

To explain the differences in memory access time we pick the data points for ptmalloc 2 and scalloc at $x=60 \%$ where the difference in memory access time is most significant and compare the number of all hardware events obtainable using perf $^{7}$. While most numbers are similar we identify two events where the numbers differ significantly. First, the L1 cache miss rate with ptmalloc 2 is $20.8 \%$ while scalloc causes a

\footnotetext{
${ }^{7}$ See https://perf.wiki.kernel.org
} 


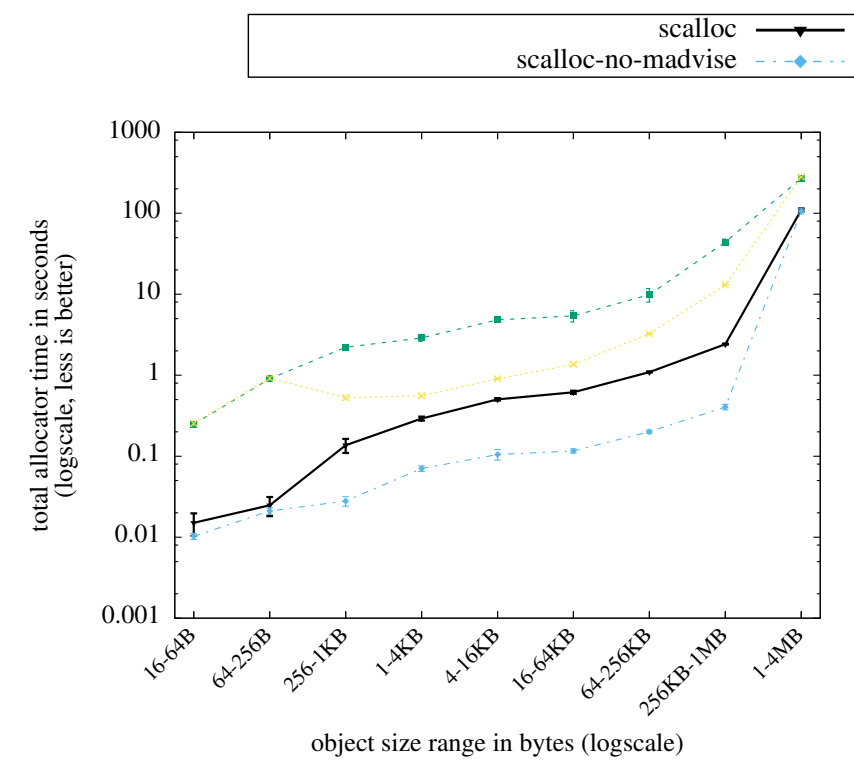

(a) Total allocator time

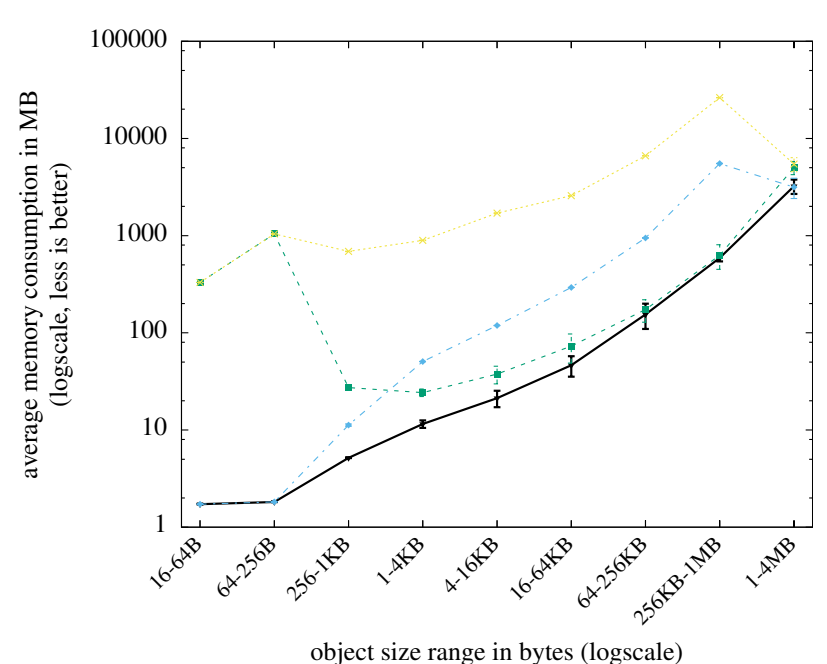

(b) Average memory consumption

Figure 12: Temporal and spatial performance for the virtual span evaluation

L1 miss rate of $15.2 \%$ at almost the same number of total L1 cache loads. We account this behavior to more effective cache line prefetching because we observe $272.2 \mathrm{M} \mathrm{L} 1$ cache prefetches with scalloc and only $119.8 \mathrm{M} \mathrm{L1}$ cache prefetches with ptmalloc2. Second, and related to the L1 miss rate, we observe 408.5M last-level cache loads with ptmalloc2 but only 190.2M last-level cache loads with scalloc because every L1 cache miss causes a cache load on the next level. The last-level cache miss rate is negligible with both allocators suggesting that the working set (by design) fits into the lastlevel cache. Note that last-level cache events in perf include L2 and L3 cache events on our hardware.

\subsection{Design Decisions}

We now evaluate scalloc's design and how the three main contributions, virtual spans, the scalable backend, and the constant-time frontend influence temporal and spatial performance. To do so, we provide several different configurations of scalloc and the benchmarking environment and compare them against each other in isolated settings in best-effort manner. By best-effort we mean that we design our experiments so that they highlight the contribution of an isolated component to overall performance. Still, frontened evaluations will also include the backend and vice versa, and disabling virtual spans is implicit via disabling a kernel feature.

\subsubsection{Virtual Spans}

Virtual spans enable uniform treatment of objects across a large range of different sizes while avoiding physical memory fragmentation through on-demand paging and explicit madvise calls. The following experiment aims at illustrat- ing the benefits, cost, and limitations of virtual spans and ultimately the role played by the paging mechanism of the operating system.

We compare four scalloc configurations on the same workload as in Section 7.5, see Figure 10a and Figure 10b. The first configuration, scalloc, is the default configuration used in all previous experiments. The second configuration, scallocno-madvise, only differs in the policy for returning memory to the operating system by disabling all madvise calls. Both configurations are executed with transparent huge pages disabled in the Linux kernel, i.e., virtual memory is mapped to $4 \mathrm{~KB}$ pages only. The third and fourth configuration are the same as scalloc and scalloc-no-madvise but with transparent huge pages enabled, i.e., the kernel may switch from $4 \mathrm{~KB}$ pages to $2 \mathrm{MB}$ pages, effectively disabling the advantages of virtual spans. Therefore we suffix those configurations with no-virtual-spans.

Figure 12a shows the total allocator time for an increasing range of object sizes. The difference between scalloc and scalloc-no-madvise is explained by the cost and consequences of calling madvise which causes consecutive page faults requiring the operating system to zero pages. However, the memory consumption of scalloc, illustrated in Figure 12b, improves almost proportionaly to the cost of calling madvise. For small objects up to 256 bytes there are no differences because madvise is only called on spans of larger size classes. For huge objects (larger than 1MB) there are no differences because scalloc allocates huge objects directly through mmap.

Enabling transparent huge pages (for scalloc-no-virtualspans and scalloc-no-madvise-no-virtual-spans) allows the kernel to allocate $2 \mathrm{MB}$ pages instead of $4 \mathrm{~KB}$ pages. Since 
the cost of zeroing $2 \mathrm{MB}$ pages is significantly higher than that of $4 \mathrm{~KB}$ pages the cost of allocating spans goes up, especially for small size classes (less than 256 bytes) where the real span size is small compared to the virtual span size. Figure 12a shows that virtual spans perform better with $4 \mathrm{~KB}$ pages than with $2 \mathrm{MB}$ pages. The memory consumption in Figure 12b increases dramatically for small size classes because the whole virtual span size is materialized (but still unused) in physical memory. For larger objects, calling madvise causes the kernel to fall back from $2 \mathrm{MB}$ pages to $4 \mathrm{~KB}$ pages. As a consequence, the scalloc-no-virtualspans configuration approaches the small memory footprint of scalloc. Still the cost of zeroing $2 \mathrm{MB}$ pages and eventually falling back to $4 \mathrm{~KB}$ pages causes a significant temporal overhead compared to scalloc. Disabling the advantages of virtual spans by enabling transparent huge pages and also disabling madvise calls (scalloc-no-madvise-no-virtualspans) causes the highest memory consumption because unused space in virtual spans is materialized in physical memory and because the kernel cannot fall back to $4 \mathrm{~KB}$ pages. The total allocator time is lower compared to scallocno-virtual-spans because no madvise calls are necessary.

From the results of this experiment we conclude that the paging mechanism of Linux is sufficient for implementing virtual spans efficiently if paging happens on a $4 \mathrm{~KB}$ granularity. We also conclude that calling madvise can trade speed for memory consumption and choosing either setting depends on the application of scalloc. However, improving the performance of madvise on operating system level would improve scalloc's virtual span design even further.

\subsubsection{Scalable Backend}

A consequence of the design around virtual and real spans is that, depending on the workload and in particular on object sizes, the backend may be subject to high levels of contention. The following experiment evaluates the design of the spanpool in terms of performance and scalability, i.e., it shows that multiple stacks per size class are necessary to deal with workloads that cause high contention on the backend. Note that for workloads that only utilize small objects, e.g. 8-byte objects, real spans provide enough buffering only requiring a single stack per size class in the span-pool. We omit separate plots for this data. However, for larger objects, real spans only provide a limited amount of blocks requiring a fast and scalable backend. For example, for 256-byte objects the real span size of $32 \mathrm{~KB}$ amounts to 127 blocks, potentially resulting in frequent interaction with the span-pool.

The experiment is based on threadtest and configured as in Section 7.2 with the only difference being that the benchmark is configured to allocate and deallocate 256-byte objects. We compare our default scalloc version, which is based on a span-pool with as many stacks per size class as threads, with a version that only uses a single stack per size class, called scalloc-no-span-pool.
Figure 13a illustrates that for an increasing number of threads, a single stack is unable to deal with contention on the span-pool, effectively resulting in degraded performance. However, in terms of memory consumption, Figure 13b shows that using a single stack results in better memory utilization as threads synchronize on a single source of empty spans. Scalloc's design trades the better performance (up to $2.7 x$ ) for slightly worse memory utilization (up to $15 \%$ ).

\subsubsection{Constant-time Frontend}

In a similar spirit, we evaluate the performance impact of a constant-time frontend that returns empty spans to the backend as soon as possible, i.e., upon freeing the last object contained in a span.

The experiment is based on threadtest and configured as in Section 7.2, see Figure 6a and Figure 6b. As the experiment is configured for small object sizes, returning memory to the operating system only plays a minor role. We compare our default scalloc version with a scalloc version that returns empty spans to the backend at a later point in time in its allocation slow path, called scalloc-reclaim-span-in-allocation. Note that threadtest provides no information about per-operation latency and hence the experiment only shows throughput and scalability, but since each thread implements a closed system, throughput is indirectly proportional to latency.

Figure 14a illustrates performance and scalability. Both scalloc versions provide almost the same speedup but differ in memory consumption illustrated in Figure 14b. The results indicate that eagerly returning spans as soon as they get empty rather than delaying reclamation until the next (slow path) allocation increases the potential for reusing spans by other threads effectively reducing memory consumption. At 39 threads the difference in memory consumption is about $25 \%$ between the two scalloc versions.

\section{Conclusion}

We presented three contributions: (a) virtual spans that enable uniform treatment of small and big objects; (b) a fast and scalable backend leveraging newly developed global data structures; and (c) a constant-time (modulo synchronization) frontend that eagerly returns empty spans to the backend. Our experiments show that scalloc is either better (threadtest, object sizes, producer-consumer) or competitive (shbench, larson, mutator locality, SPEC) in performance and memory consumption compared to other allocators.

To conclude, the problem of high-performance and scalable memory allocation is complex. There may be different solutions. Our solution was guided by an initial idea to design an allocator whose scalability benefits from the scalability of recently developed concurrent data structures. In order to make maximal use of global data structures, we developed virtual spans and additionally the constant-time frontend. It may be interesting to study other applications of global scalable concurrent data structures. 


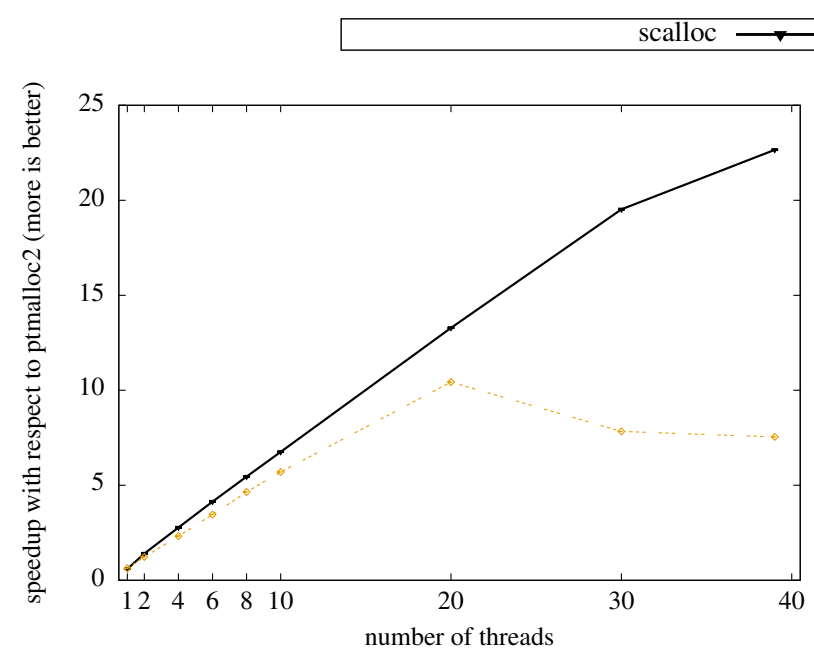

(a) Speedup

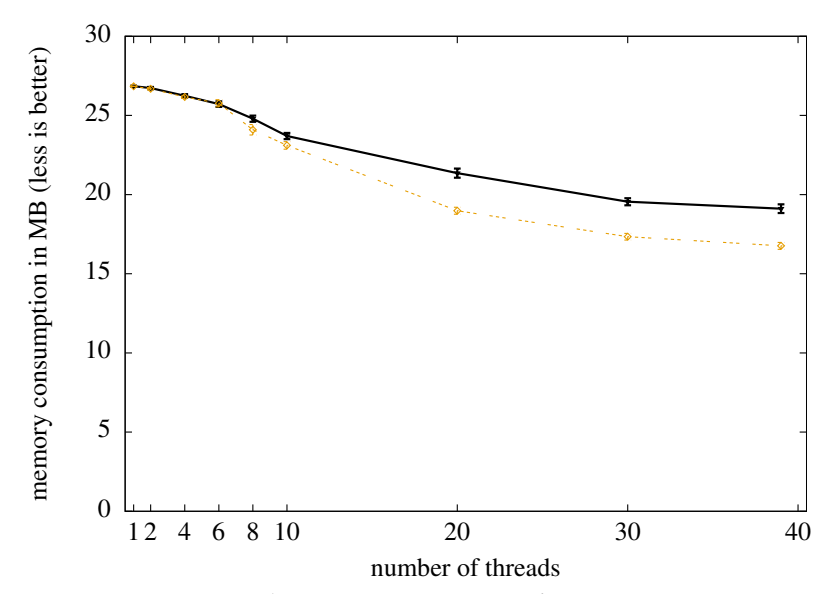

(b) Memory consumption

Figure 13: Span-Pool evaluation: Threadtest benchmark using 256-byte objects

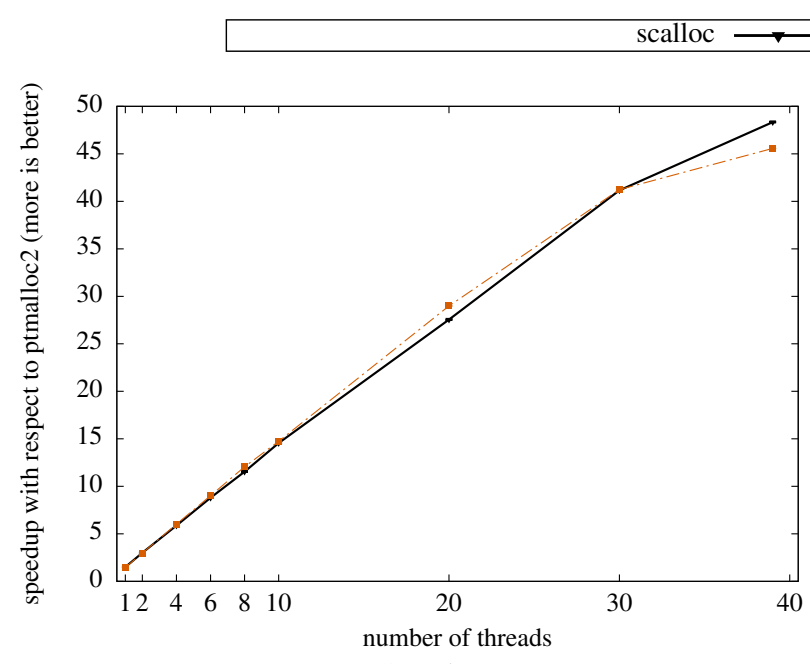

(a) Speedup

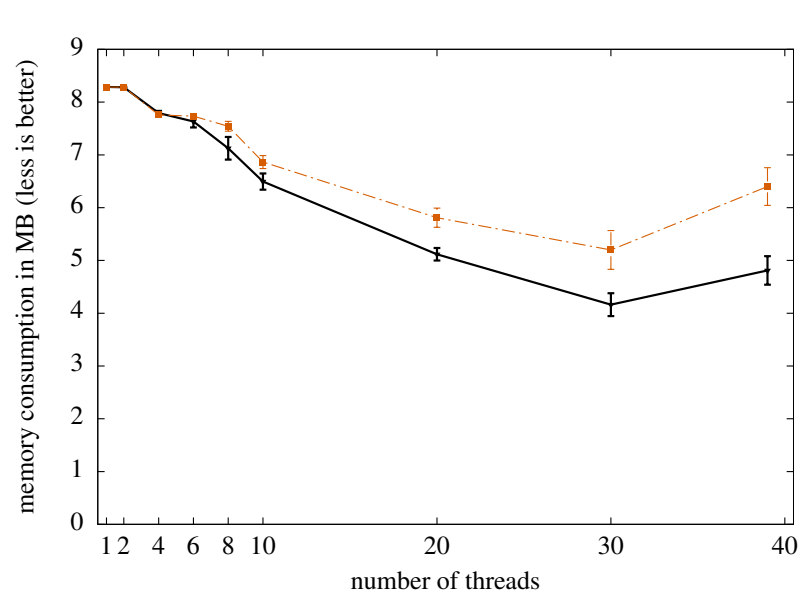

(b) Memory consumption

Figure 14: Frontend evaluation: Threadtest benchmark using 64-byte objects

Other interesting future work may be (1) integrating virtual spans and virtual memory management, (2) NUMA-aware mapping of real spans, and in particular (3) dynamically resizing real spans to trade off $\mathrm{LAB}$ provisioning and performance based on run-time feedback from mutators, similar in spirit to just-in-time optimizations in virtual machines.

\section{Acknowledgements}

This work has been supported by the National Research Network RiSE on Rigorous Systems Engineering (Austrian Science Fund (FWF): S11404-N23, S11411-N23) and a Google PhD Fellowship.

\section{References}

[1] Y. Afek, G. Korland, and E. Yanovsky. Quasi-linearizability: Relaxed consistency for improved concurrency. In Proc. Conference on Principles of Distributed Systems (OPODIS), pages 395-410. Springer, 2010. doi: 10.1007/978-3-642-17653-1_ 29.

[2] M. Aigner and C. Kirsch. ACDC: Towards a universal mutator for benchmarking heap management systems. In Proc. International Symposium on Memory Management (ISMM), pages 75-84. ACM, 2013. doi: 10.1145/2464157.2464161. 
[3] E. Berger, K. McKinley, R. Blumofe, and P. Wilson. Hoard: a scalable memory allocator for multithreaded applications. In Proc. International Conference on Architectural Support for Programming Languages and Operating Systems (ASPLOS), pages 117-128. ACM, 2000. doi: 10.1145/384264.379232.

[4] E. Berger, B. Zorn, and K. McKinley. Reconsidering custom memory allocation. In Proc. Conference on Object-oriented Programming, Systems, Languages, and Applications (OOPSLA), pages 1-12. ACM, 2002. doi: 10.1145/582419.582421.

[5] S. Blackburn, P. Cheng, and K. McKinley. Oil and water? High performance garbage collection in Java with MMTk. In Proc. International Conference on Software Engineering (ICSE). IEEE, 2004. doi: 10.1109/ICSE.2004.1317436.

[6] A. Clements, M. Kaashoek, and N. Zeldovich. RadixVM: Scalable address spaces for multithreaded applications. In Proc. ACM European Conference on Computer Systems (EuroSys), pages 211-224. ACM, 2013. doi: 10.1145/2465351.2465373.

[7] M. Dodds, A. Haas, and C. Kirsch. A scalable, correct time-stamped stack. In Proc. Symposium on Principles of Programming Languages (POPL), pages 233-246. ACM, 2015. doi: 10.1145/2775051.2676963.

[8] J. Evans. A scalable concurrent malloc(3) implementation for freebsd. In Proc. BSDCan, 2006.

[9] W. Gloger. ptmalloc2 - a multi-thread malloc implementation. http://malloc.de/en/.

[10] Google Inc. gperftools: Fast, multi-threaded malloc() and nifty performance analysis tools. http://code.google.com/p/ gperftools/.

[11] A. Haas, T. Henzinger, C. Kirsch, M. Lippautz, H. Payer, A. Sezgin, and A. Sokolova. Distributed queues in shared memory-multicore performance and scalability through quantitative relaxation. In Proc. International Conference on Computing Frontiers $(C F)$, pages 17:1-17:9. ACM, 2013. doi: 10.1145/2482767.2482789.

[12] A. Haas, T. Henzinger, A. Holzer, C. Kirsch, M. Lippautz, H. Payer, A. Sezgin, A. Sokolova, and H. Veith. Local linearizability. CoRR, abs/1502.07118, 2015.

[13] D. Hendler, I. Incze, N. Shavit, and M. Tzafrir. Flat combining and the synchronization-parallelism tradeoff. In Proc. Symposium on Parallel Algorithms and Architectures (SPAA), pages 355-364. ACM, 2010. doi: 10.1145/1810479.1810540.

[14] J. L. Henning. Spec cpu2006 benchmark descriptions. SIGARCH Comput. Archit. News, 34(4):1-17, 2006. doi: 10.1145/1186736.1186737.

[15] T. Henzinger, C. Kirsch, H. Payer, A. Sezgin, and A. Sokolova. Quantitative relaxation of concurrent data structures. In Proc. Symposium on Principles of Programming Languages (POPL), pages 317-328. ACM, 2013. doi: 10.1145/2429069.2429109.
[16] M. Herlihy and N. Shavit. The Art of Multiprocessor Programming. Morgan Kaufmann Publishers Inc., 2008.

[17] R. Hudson, B. Saha, A.-R. Adl-Tabatabai, and B. Hertzberg. Mcrt-malloc: a scalable transactional memory allocator. In Proc. International Symposium on Memory Management (ISMM), pages 74-83. ACM, 2006. doi: 10.1145/1133956. 1133967.

[18] Intel Corporation. Thread building blocks (tbb). http: //threadingbuildingblocks.org.

[19] A. Kogan and E. Petrank. A methodology for creating fast waitfree data structures. In Proc. Symposium on Principles and Practice of Parallel Programming (PPoPP), pages 141-150. ACM, 2012. doi: 10.1145/2145816.2145835.

[20] B. Kuszmaul. Supermalloc: A super fast multithreaded malloc for 64-bit machines. In Proc. International Symposium on Memory Management (ISMM), pages 41-55. ACM, 2015. doi: 10.1145/2754169.2754178.

[21] P.-A. Larson and M. Krishnan. Memory allocation for longrunning server applications. In Proc. International Symposium on Memory Management (ISMM), pages 176-185. ACM, 1998. doi: $10.1145 / 286860.286880$.

[22] D. Lea. A memory allocator. http://g.oswego.edu/dl/ html/malloc.html.

[23] Lockless Inc. 1lalloc: Lockless memory allocator. http: //locklessinc.com/.

[24] M. Michael. Hazard pointers: Safe memory reclamation for lock-free objects. IEEE Trans. Parallel Distrib. Syst., 15(6): 491-504, 2004. doi: 10.1109/TPDS.2004.8.

[25] M. Michael. Scalable lock-free dynamic memory allocation. In Proc. Conference on Programming Language Design and Implementation (PLDI), pages 35-46. ACM, 2004. doi: 10. 1145/996893.996848.

[26] MicroQuill Inc. shbench. http://www . microquill.com/.

[27] S. Schneider, C. Antonopoulos, and D. Nikolopoulos. Scalable locality-conscious multithreaded memory allocation. In Proc. International Symposium on Memory Management (ISMM), pages 84-94. ACM, 2006. doi: 10.1145/1133956.1133968.

[28] K. Serebryany, D. Bruening, A. Potapenko, and D. Vyukov. Addresssanitizer: A fast address sanity checker. In Proc. USENIX Conference on Annual Technical Conference (USENIX ATC), pages 28-28. USENIX Association, 2012.

[29] R. Treiber. Systems programming: Coping with parallelism. Technical Report RJ-5118, IBM Research Center, 1986.

[30] P. Wilson, M. Johnstone, M. Neely, and D. Boles. Dynamic storage allocation: A survey and critical review. In Proc. International Workshop on Memory Management (IWMM), pages 1-116. Springer, 1995. doi: 10.1007/3-540-60368-9_19. 